\title{
Star formation and mass assembly in high redshift galaxies ${ }^{\star}$
}

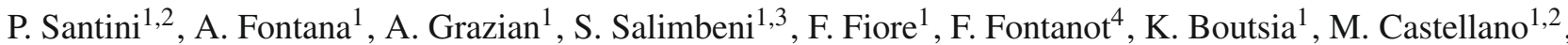 \\ S. Cristiani ${ }^{5}$, C. De Santis ${ }^{6,7}$, S. Gallozzi $^{1}$, E. Giallongo ${ }^{1}$, N. Menci ${ }^{1}$, M. Nonino ${ }^{5}$, D. Paris ${ }^{1}$, \\ L. Pentericci ${ }^{1}$, and E. Vanzella ${ }^{5}$
}

1 INAF - Osservatorio Astronomico di Roma, via Frascati 33, 00040 Monteporzio (RM), Italy e-mail: santini@oa-roma.inaf.it

2 Dipartimento di Fisica, Università di Roma "La Sapienza", P.le A. Moro 2, 00185 Roma, Italy

3 Department of Astronomy, University of Massachusetts, 710 North Pleasant Street, Amherst, MA 01003, USA

4 MPIA Max-Planck-Institute für Astronomie, Koenigstuhl 17, 69117 Heidelberg, Germany

5 INAF - Osservatorio Astronomico di Trieste, via G.B. Tiepolo 11, 34131 Trieste, Italy

${ }^{6}$ Dip. di Fisica, Università Tor Vergata, via della Ricerca Scientifica 1, 00133 Roma, Italy

7 INFN - Roma Tor Vergata, via della Ricerca Scientifica 1, 00133 Roma, Italy

Received 27 November 2008 / Accepted 24 April 2009

\begin{abstract}
Aims. The goal of this work is to infer the star formation properties and the mass assembly process of high redshift $(0.3 \leq z<$ 2.5) galaxies from their IR emission using the $24 \mu \mathrm{m}$ band of MIPS-Spitzer.

Methods. We used an updated version of the GOODS-MUSIC catalog, which has multiwavelength coverage from 0.3 to $24 \mu \mathrm{m}$ and either spectroscopic or accurate photometric redshifts. We describe how the catalog has been extended by the addition of mid-IR fluxes derived from the MIPS $24 \mu \mathrm{m}$ image. We compared two different estimators of the star formation rate (SFR hereafter). One is the total infrared emission derived from $24 \mu \mathrm{m}$, estimated using both synthetic and empirical IR templates. The other one is a multiwavelength fit to the full galaxy SED, which automatically accounts for dust reddening and age-star formation activity degeneracies. For both estimates, we computed the SFR density and the specific SFR.

Results. We show that the two SFR indicators are roughly consistent, once the uncertainties involved are taken into account. However, they show a systematic trend, IR-based estimates exceeding the fit-based ones as the star formation rate increases. With this new catalog, we show that: $a$ ) at $z>0.3$, the star formation rate is correlated well with stellar mass, and this relationship seems to steepen with redshift if one relies on IR-based estimates of the SFR; $b$ ) the contribution to the global SFRD by massive galaxies increases with redshift up to $\simeq 2.5$, more rapidly than for galaxies of lower mass, but appears to flatten at higher $z ; c$ ) despite this increase, the most important contributors to the SFRD at any $z$ are galaxies of about, or immediately lower than, the characteristic stellar mass; d) at $z \simeq 2$, massive galaxies are actively star-forming, with a median $S F R \simeq 300 M_{\odot} \mathrm{yr}^{-1}$. During this epoch, our targeted galaxies assemble a substantial part of their final stellar mass; $e$ ) the specific SFR (SSFR) shows a clear bimodal distribution.

Conclusions. The analysis of the SFR density and the SSFR seems to support the downsizing scenario, according to which high mass galaxies have formed their stars earlier and more rapidly than their low mass counterparts. A comparison with renditions of theoretical simulations of galaxy formation and evolution indicates that these models follow the global increase in the SSFR with redshift and predict the existence of quiescent galaxies even at $z>1.5$. However, the average SSFR is systematically underpredicted by all models considered.
\end{abstract}

Key words. galaxies: evolution - galaxies: high-redshift - galaxies: fundamental parameters - galaxies: photometry galaxies: starburst

\section{Introduction}

Answering the basic questions about the birth, formation, mass build-up, and evolution of galaxies throughout cosmic time are some of the major goals of observational extragalactic astronomy.

In the past few years, this issue has been approached with two different methods. Many previous works measured a rapid evolution in the stellar mass density between $z \sim 1$ and $z \sim 3$ (Dickinson et al. 2003; Fontana et al. 2003, 2004; Glazebrook et al. 2004; Drory et al. 2004; Fontana et al. 2006;

^ GOODS-MUSIC multiwavelength photometric catalog is available in electronic form at the CDS via anonymous ftp to

cdsarc.u-strasbg. fr (130.79.128.5) or via

http://cdsweb.u-strasbg.fr/cgi-bin/qcat?J/A+A/504/751
Rudnick et al. 2006; Papovich et al. 2006; Yan et al. 2006; Pozzetti et al. 2007) and demonstrated that a substantial fraction (30-50\%) of the stellar mass formed during this epoch. The differential evolution in the galaxy stellar mass function, according to which massive galaxies evolve rapidly up to $z \sim 1.5$ and then more gradually until the present epoch, while less massive galaxies continue to evolve, implies that massive galaxies must have already formed by $z \sim 1.5$. Several groups (e.g., Faber et al. 2007; Brown et al. 2007) studied the evolution of massive galaxies at $z \lesssim 1$, and their migration from the blue cloud to the red sequence. We note that optical observations (Bell et al. 2004; Zucca et al. 2006) suggest that the number of massive galaxies, as well as the stellar mass on the red sequence, has nearly doubled since $z \sim 1$, in qualitative agreement with the hierarchical merging scenario. 
A parallel line of study has analyzed the rate at which galaxies form stars during different epochs and shown that they experience an extremely active phase in the same redshift range (e.g., Lilly et al. 1996; Madau et al. 1996; Steidel et al. 1999; Hopkins 2004; Hopkins \& Beacom 2006; Daddi et al. 2007b). Galaxies appear to form their stars following the so-called downsizing scenario, in which the star formation shifts from high mass to low mass galaxies as redshift decreases. This picture was first introduced by Cowie et al. (1996), who studied the evolution in the $K_{\mathrm{s}}$ band luminosity function with redshift, and proposes that the most massive galaxies assemble their mass both earlier and more quickly than their lower mass counterparts, which, in contrast, continue to form stars until recent epochs. Later on, many other groups (e.g., Brinchmann \& Ellis 2000; Fontana et al. 2003; Feulner et al. 2005; Pérez-González et al. 2005; Papovich et al. 2006; Damen et al. 2009) derived confirmations of a downsizing behaviour from the study of the specific star formation rate, defined to be the star formation rate per unit mass, at different redshifts. However, we note that deep radio observations (Dunne et al. 2009) appear to conflict with this scenario. The downsizing picture also appears to contradict the hierarchical growth scenario in which the most massive structures that we see today are produced by merging processes between smaller structures inside large-scale overdensities and collapsed when the Universe was far younger than today.

To reproduce this early formation of massive galaxies (see Thomas et al. 2005) that are already "red and dead" at high $z$, theoretical models had to introduce very efficient processes of star formation and its suppression by means of active galactic nuclei and supernovae quenching of cooling flows (Menci et al. 2006; Kitzbichler \& White 2007; Bower et al. 2006; Croton et al. 2006; Nagamine et al. 2006; Monaco et al. 2007), gravitational heating (Khochfar \& Ostriker 2008; Johansson et al. 2009), or shock heating (Dekel \& Birnboim 2006). These models differ slightly in their predictions mainly because they adopt different processes to shut down the star formation.

Both stellar masses and star formation rate estimates are affected by a number of uncertainties. The measure of the star formation rate (SFR) is especially difficult to handle. The high amount of energy produced by newly born stars is emitted throughout the galaxy spectral energy distribution (SED), from $\mathrm{X}$-rays to radio frequencies. Since we are of course unable to directly measure the total light emitted by young and massive stars, calibration factors and corrections are applied to estimate its value for any of these frequency ranges (Kennicutt 1998; Bell 2003; Calzetti 2008). One of the most commonly used estimators is the UV rest-frame band, where young and massive stars emit most of their light. However, dust absorbs, reprocesses, and re-radiates UV photons at near-to-far IR wavelengths. Hence, the reliability of UV luminosity as a SFR tracer depends on large and uncertain corrections relying upon the dust properties, which are not yet clearly known (Calzetti et al. 1994; Calzetti 1997, 2001). Moreover, the UV-upturn at $\lambda$ shortward of $2500 \AA$ (e.g., Han et al. 2007), especially in elliptical galaxies, can potentially bias the SFR estimate at very low redshift. Since the most intense star formation episodes are expected to occur in dusty regions, most of the power originating in star-forming (SF) galaxies is emitted in this wavelength range, and the dust emission peak is the dominant component of SF galaxies SEDs (Adelberger \& Steidel 2000; Calzetti et al. 2000). Thus, a popular approach consists of adopting a conversion between the total emitted IR luminosity $\left(L_{\mathrm{IR}}\right.$ hereafter) and a star formation rate estimation that is unaffected by dust obscuration (Kennicutt 1998).
The total infrared luminosity is generally estimated by comparing observed SEDs and synthetic templates, although empirical conversions have sometimes been used (Takeuchi et al. 2005; Bavouzet et al. 2008). A variety of different libraries are used for this purpose (e.g., Chary \& Elbaz 2001; Dale \& Helou 2002; Lagache et al. 2003; Siebenmorgen \& Krügel 2007, and so on). A notable problem for the reliability of IR-based SFR tracers concerns obscured AGNs. In these objects the IR emission is generated by matter accretion onto a central black hole rather than dust heating by young stars.

In this paper, we use the GOODS-MUSIC catalog to investigate properties of star-forming galaxies up to redshift 2.5 and infer the mass assembly process from their mid-IR emission. The paper is organized as follows. In Sect. 2, we recall the basic features of the GOODS-MUSIC dataset and explain the innovations concerning its latest version, and we explain how it has been updated with the addition of the $24 \mu \mathrm{m}$ photometric band. We derive and compare star formation rates from IR- and fit-based estimators in Sect. 3. In Sect. 4, we present a study of the mass assembly process in the high redshift Universe and a comparison with theoretical model predictions. We finally summarize our work and our conclusions in Sect. 5. In Appendix A, we describe in more detail how we convert mid-IR fluxes into total infrared luminosities and compare the different templates used, and in Appendix B we present the error analysis performed on the fit-based SFR estimates.

Throughout this work, unless stated otherwise, we assume a Salpeter (1955) initial mass function (IMF) and adopt the $\Lambda$-CDM concordance cosmological model $\left(H_{0}=\right.$ $70 \mathrm{~km} \mathrm{~s}^{-1} \mathrm{Mpc}^{-1}, \Omega_{\mathrm{M}}=0.3$ and $\left.\Omega_{\Lambda}=0.7\right)$.

\section{The data sample}

\subsection{The new GOODS-MUSIC sample}

We present and use an updated version of the multicolour GOODS-MUSIC sample (GOODS MUlticolour Southern Infrared Catalog, Grazian et al. 2006), extracted from the public data of the GOODS-South survey (Giavalisco et al. 2004). In the following, we shall refer to this version of the catalog as GOODS-MUSIC v2, to differentiate it from the former public version, which is named $\mathrm{v} 1$ hereafter. The new version is also made publicly available ${ }^{1}$.

The 15-bands multiwavelength coverage ranges from 0.35 to $24 \mu \mathrm{m}$, as a result of the combination of images from different instruments (2.2ESO, VLT-VIMOS, ACS-HST, VLTISAAC, Spitzer-IRAC, Spitzer-MIPS). The catalog covers an area of $\sim 143.2 \mathrm{arcmin}^{2}$ located in the Chandra Deep Field South and consists of 15208 sources. After culling Galactic stars, it contains 14999 objects selected in either the $z$ band or the $K_{\mathrm{s}}$ band or at $4.5 \mu \mathrm{m}$.

The whole catalog has been cross-correlated with spectroscopic catalogs available to date, and a spectroscopic redshift has been assigned to $\sim 12 \%$ of all sources. For all other objects, we have computed well-calibrated photometric redshifts using a standard $\chi^{2}$ minimization technique for a large set of synthetic spectral templates.

The previous version of the catalog and procedures adopted to determine the photometric redshifts and physical properties of each object were described at length in Grazian et al. (2006)

\footnotetext{
1 The catalog is available in electronic form at the CDS. It is also possible to download the catalog at the WEB site http: //lbc . mporzio. astro.it/goods.
} 
and Fontana et al. (2006). With respect to the previous catalog, we have performed a set of improvements to the optical-near-IR data, the major ones being:

- In addition to objects selected in the ACS $z$ and in the ISAAC $K_{\mathrm{S}}$ bands, we have also included objects selected from the IRAC $4.5 \mu \mathrm{m}$ image, hence including sources detected at $4.5 \mu \mathrm{m}$ but very faint or undetected even in $K_{\mathrm{s}}$ band. A full description of these objects is beyond the scope of the present paper and will be presented elsewhere.

- We have revised the photometry in the four IRAC bands using an updated version of the PSF-matching kernels, as released by the Spitzer Science Center. As a consequence, we also adopted a larger kernel, to fully account for the large tails of the IRAC PSFs.

- We have adopted a revised procedure for estimating the background in the IRAC images. Using the objects' positions and IRAC fluxes from the GOODS-MUSIC v1 catalog, we have created realistic simulated images in the four IRAC bands by smoothing sources to the nominal IRAC PSFs. An accurate background estimation has been performed by subtracting these simulated images from the original ones and by linearly interpolating the residual emission. Since the average value of the apparent IRAC background is negative, this has led to an increase in the adopted background, with respect to the GOODS-MUSIC v1 version.

- These two changes have modified the IRAC photometry. Because of the new kernels, the brightest objects have a higher flux and a typical offset in magnitude of $0.23,0.14$, $0.22,0.35$, respectively in the $3.6,4.5,5.8$, and $8 \mu \mathrm{m}$ images, with respect to the GOODS-MUSIC v1 catalog. The new photometry agrees with the analysis of Wuyts et al. (2008). This effect is largely mitigated for fainter galaxies, since the higher background now adopted has led to an underestimate of their fluxes.

- Overall, the revised IRAC photometry has a modest impact on the estimate of photometric redshifts, since $\left\langle z_{\text {phot }} v 2-\right.$ $\left.z_{\text {phot } v 1}\right\rangle \sim 0.01 \pm 0.16$ for the whole sample and $\sim 0.01 \pm 0.03$ when a $3 \sigma$-clipping analysis is performed (see also Wuyts et al. 2008).

- A more informative test of the photometric redshift accuracy comes from the enlargement of the sample of galaxies with spectroscopic redshifts, which we obtained by adding new spectra from public surveys (Vanzella et al. 2008; Popesso et al. 2009). In addition, we have also had access to the spectra of the GMASS survey (Cimatti et al. 2008), prior to their publication. The final sample now includes 1888 galaxies, three times larger than the spectroscopic sample in Grazian et al. (2006). The additional spectra are mostly relative to galaxies that are both fainter and at higher redshift than in the original sample. Without significant refinements in the adopted templates, we then find that the absolute scatter $|\Delta z|=\left|z_{\text {spe }}-z_{\text {phot }}\right| /\left(1+z_{\text {spe }}\right)$ has a slightly larger average value. Quantitatively, the average absolute scatter is now $\langle|\Delta z|\rangle=0.06$, instead of 0.045 obtained for the GOODSMUSIC v1 catalog. However, when only the brightest galaxies are considered, we find comparable values with respect to Grazian et al. (2006) $(\langle|\Delta z|\rangle=0.043)$. We have verified that this is due to an increased number of outliers, as shown by a $3 \sigma$-clipping analysis, which provides $\langle|\Delta z|\rangle=0.027$ and 0.032 for the complete datasets of v1 and v2 catalogs, respectively.

- We have removed Galactic stars and performed a more careful selection of the galaxy sample to identify AGN sources.
For the latter, we have first removed all objects whose spectra show AGN features. Then, we have cross-correlated our catalog with the X-ray catalog of Brusa et al. (2009), and removed all X-ray detected sources whose flux is dominated by an unresolved central source. These sources typically have spectra classified as narrow-line AGNs. The optical morphologies of all remaining X-ray sources do not show a dominating central point like source, and - where available - have typically spectra classified as emission-line starforming galaxies. These objects have been retained in our galaxy sample.

The major new ingredient of this new version of the GOODSMUSIC catalog, however, is the inclusion of the $24 \mu \mathrm{m}$ photometry for all galaxies in the sample, which is the main focus of the present paper. We describe the adopted procedures and results in the following.

\subsection{MIPS $24 \mu \mathrm{m}$ catalog}

We have extended the GOODS-MUSIC catalog with the addition of the mid-IR fluxes derived from the public $24 \mu \mathrm{m}$ image of the Multiband Imager Photometer for Spitzer (MIPS, Rieke et al. 2004) onboard the Spitzer Space Telescope. As for the IRAC images, given the very large PSF of this image ( $\sim 5.2 \operatorname{arcsec})$, to properly detect and de-blend objects we had to employ a PSF-matching technique, which is performed by the software ConvPhot (De Santis et al. 2007). This algorithm measures colours from two images of different qualities by exploiting the spatial and morphological information contained in the higher resolution image.

When applying ConvPhot to our case, each object was extracted from the high resolution $z$ band ACS-HST (PSF $\sim 0.12^{\prime \prime}$ ) image, which was used as a prior to extract the objects' positions, filtered with a convolution kernel, and finally scaled by a $\chi^{2}$ minimization over all the image pixels to match the intensity in the MIPS image. To fully use the positional information of the ACS images, and maintain consistency with the $z$-selected sample, we used the $z$ band ACS-HST data as a prior, augmented by artificial objects placed where galaxies detected only in $K_{\mathrm{S}}$ or $4.5 \mu \mathrm{m}$ were located. In our case, the MIPS-Spitzer and ACSHST images have pixel scales of 1.2 and $0.03 \mathrm{arcsec} / \mathrm{pixel}$, which means that it is impracticable to use ConvPhot even with fast workstations. To make the computation feasible, we rebinned the ACS detection image by a factor $8 \times 8(0.24 \mathrm{arcsec} /$ pixel $)$. In regions where the crowding of the $z$-detected sources was significant, the fit may become unconstrained or degenerate because of the large size of the MIPS PSF. To prevent this, we placed an additional constraint on the fitted fluxes that must be non-negative. For the objects whose flux is forced to be zero, we provide an upper limit derived from the analysis of the mean rms in the object area.

The behaviour of ConvPhot in these extreme applications was tested with several simulations, which were described by De Santis et al. (2007). These tests indicated that the estimated magnitudes are biased by neither the different qualities of the two images nor the undersampling of the high resolution image after rebinning.

Finally, from visual inspection of sources with unusual colours, we removed $\sim 30$ objects from the catalog whose flux was incorrectly assigned. By examination of the residuals, we also verified that there is no significantly bright source in the MIPS image apart from the ones considered. We detected 3313 


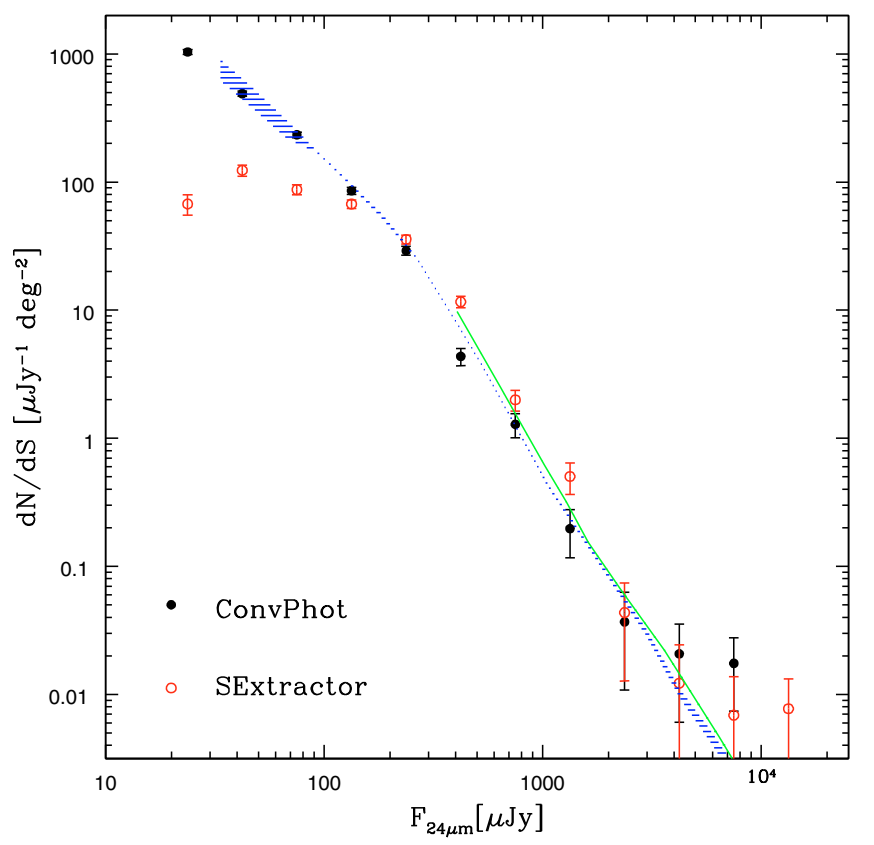

Fig. 1. $24 \mu \mathrm{m}$ flux number counts measured by ConvPhot (black filled circles) and SExtractor (red empty circles). Error bars have been computed as the square root of the number of objects in each bin. We compared our results to Papovich et al. (2004) and Shupe et al. (2008) $24 \mu \mathrm{m}$ counts (blue shaded region and green line, respectively).

\section{( $\sim 22 \%$ of total) detected objects and $11841(\sim 78 \%) 1 \sigma$ upper} limits.

Despite the validation tests providing satisfying results, we emphasize that the intrinsic limitations due to the poor resolution of the MIPS image cannot be completely overcome, in particular for sources blended even in the detection image, i.e., sources whose profiles overlap in the ACS $z$ image. In this case, the separation between the two - or more - objects can become even smaller than the positional accuracy of the MIPS images, and the association between the $z$-detected and the MIPS sources relies on the accuracy of the astrometric solution. To check for possible misidentifications, we associated each source with a flag to quantify the number of possible contaminants, at different distances. This flag was attached to the public catalog and we caution the user to check for possible systematic errors. The results that we shall present in the following are insensitive to the inclusion of the most blended sources, which have therefore not been removed.

It may be interesting to observe the effect of this procedure on the $24 \mu \mathrm{m}$ number counts, shown in Fig. 1. We present both the counts derived by ConvPhot as well as those obtained by a SExtractor catalog, which was produced by adopting corrected aperture magnitudes at 6 arcsec. The blue shaded region shows the $24 \mu \mathrm{m}$ counts from Papovich et al. (2004). They counted sources in five different fields (CDFS among them) using DAOPHOT software. The green solid line (error bars are not large enough to be seen) represents Shupe et al. (2008) counts in the SWIRE field performed by SExtractor. ConvPhot (black dots) provides consistent results with both the previous work of other authors to the lowest fluxes and our SExtractor catalog (red circles) at $F \gtrsim 100 \mu \mathrm{Jy}$.

The agreement at bright fluxes confirms the results of the simulations and validation tests presented in De Santis et al. (2007) and shows that the fluxes estimated by ConvPhot agree with those estimated by an independent detection with SExtractor for sources that are not severely blended. For the blended fraction of the objects, however, the fluxes estimated by ConvPhot can be slightly lower, since part of the flux is identified with the fainter contaminants, which are not detected in the $24 \mu \mathrm{m}$ image alone.

It is more interesting to consider the behaviour at faint fluxes, where the a priori knowledge of the object position, because of the use of the $z, K_{\mathrm{s}}$ and $4.5 \mu \mathrm{m}$ images for the detection, allows us to obtain flux estimate at much fainter limits, reducing the effects of blending and confusion. As expected, indeed, SExtractor counts decline at $\sim 100 \mu \mathrm{Jy}$, where the confusion limit prevents the detection of fainter sources, while ConvPhot allows us to complete our detection to even deeper limits. ConvPhot number counts present a double slope, with a break point located at $\sim 100 \mu \mathrm{Jy}$, which we consider to be an intrinsic property of the sample. The slope and the normalization at the faint end agree with the estimates of Papovich et al. (2004), who carefully computed a correction for the incompleteness due to poor resolution at faint limits. Papovich et al. (2004) and subsequent papers (e.g., Le Floc'h et al. 2005; Pérez-González et al. 2005; Papovich et al. 2006; Marcillac et al. 2006; Bell et al. 2007) estimated that the source detection in MIPS-CDFS is $80 \%$ complete at $83 \mu \mathrm{Jy}$ or so, which typically corresponds to $S / N \sim 28-30$ in our fitting procedure. For consistency with these works, we therefore distinguish between objects with fluxes above this limit and those detected at lower $S / N$, to fluxes as low as $\sim 20 \mu \mathrm{Jy}$, which corresponds to our flux count limit. The median $S / N$ at this flux limit is $\sim 6-7$, although a tail is present at lower values of $S / N$ that is caused by source blending. Nevertheless, this tail includes only a small number of objects.

Finally, we cross-correlated our $24 \mu \mathrm{m}$ catalog with both the FIREWORKS catalog (Wuyts et al. 2008) and the one released by the GOODS Team ${ }^{2}$ (Chary 2007), who adopted a similar source extraction technique. The overall agreement was good with a small offset in both

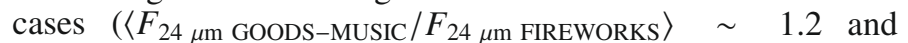
$\left.\left\langle F_{24 \mu \mathrm{m} \text { GOODS-MUSIC }} / F_{24} \mu \mathrm{m} D R 3\right\rangle \sim 0.91\right)$.

\subsection{The data selection}

As pointed out above, we removed from our catalog Galactic stars and both spectroscopic or X-ray detected AGN sources. Moreover, we only consider the redshift range $0.3-2.5$. From this sample, we consider the following two subsamples:

- the purely $K_{\mathrm{s}}$-selected sample, subsample A, consists of 2602 galaxies. Of these, 981 (376) are $24 \mu \mathrm{m}$ detections with $F_{24 \mu \mathrm{m}}>20(83) \mu \mathrm{Jy}$ and 1621 have been assigned an upper limit;

- subsample B was created by performing the following cuts: $z<26$ or $K_{\mathrm{s}}<23.5$ or $m_{4.5}<23.2$. It includes 7909 galaxies, of which 1165 (413) are $24 \mu \mathrm{m}$ detections with $F_{24 \mu \mathrm{m}}>20$ (83) $\mu \mathrm{Jy}$ and 6744 are upper limits.

\section{Comparison between SFR indicators}

In this section, we present different methods to estimate the star formation rate and compare the results obtained from the different indicators.

\footnotetext{
${ }^{2}$ GOODS-South MIPS 24 micron source list v0.91 from GOODS data release (DR3).
} 


\subsection{SFR estimators: IR-, fit- and UV-based SFR}

Based on the assumption that most of the photons originating in newly formed stars are absorbed and re-emitted by dust, the mid-IR emission is in principle the most sensitive tracer of the star formation rate. In addition, a small fraction of unabsorbed photons will be detected at UV wavelengths. A widely used SFR indicator is therefore based on a combination of IR and UV luminosity, which supplies complementary knowledge of the star formation process (Iglesias-Páramo et al. 2006; Calzetti et al. 2007). For $24 \mu \mathrm{m}$ detected sources, we estimated the instantaneous SFR using the same calibration as Papovich et al. (2007) and Bell et al. (2005):

$S F R_{\mathrm{IR}+\mathrm{UV}} / M_{\odot} \mathrm{yr}^{-1}=1.8 \times 10^{-10} \times L_{\mathrm{bol}} / L_{\odot}$

$L_{\mathrm{bol}}=\left(2.2 \times L_{\mathrm{UV}}+L_{\mathrm{IR}}\right)$.

We computed $L_{\mathrm{IR}}$ by fitting $24 \mu \mathrm{m}$ emission to Dale \& Helou (2002, DH hereafter) synthetic templates, which are widely adopted in the literature. In Appendix A, we compare these values with the corresponding $L_{\mathrm{IR}}$ predicted by different model libraries (Chary \& Elbaz 2001; Polletta et al. 2007). We include the rest-frame UV luminosity, uncorrected for extinction, derived from the SED fitting technique, $L_{\mathrm{UV}}=1.5 \times L_{2700} \AA$; although often negligible, this can account for the contribution from young unobscured stars.

Following Papovich et al. (2007), we then applied a lowering correction to the estimate obtained from Eq. (1). They found that the $24 \mu \mathrm{m}$ flux, fitted with the same DH library, overestimates the SFR for bright IR galaxies with respect to the case where longer wavelengths (70 and $160 \mu \mathrm{m}$ MIPS bands) are considered as well, and they corrected the trend using an empirical second-order polynomial. In Appendix A we show further confirmation of the need to apply this correction: for bright sources, $L_{\mathrm{IR}}$ estimated by synthetic models has values of up to a factor of 10 higher than $L_{\mathrm{IR}}$ predicted by the empirical library of Polletta et al. (2007). Similar results were also published by Bavouzet et al. (2008) and Rieke et al. (2009). In the following, we refer to the estimate in Eq. (1) as $S F R_{\mathrm{IR}+\mathrm{UV}}$.

A complementary approach to estimating the star formation rate, as well as other galaxy physical properties (e.g., mass, age, dust extinction), is the SED fitting. A grid of spectral templates is computed from standard spectral synthesis models, and the expected magnitudes in our filter set are calculated. The derived template library is compared with the available photometry and the best-fit model template was adopted according to a $\chi^{2}$ minimization. During the fitting process, the redshift is fixed to its spectroscopic or photometric value. The physical parameters associated with each galaxy are obtained from the best-fit template up to $5.5 \mu \mathrm{m}$ rest-frame. This analysis assumes that the overall galaxy SED can be represented as a purely stellar SED, extincted by a single attenuation law, and that the relevant $E(B-V)$ and basic stellar parameters (mostly age and star formation history, but also metallicity) can be simultaneously recovered with a multiwavelength fit. We note that parameter degeneracies cannot be completely removed, especially at high redshift. Previous studies (Papovich et al. 2001; Shapley et al. 2001, 2005) demonstrated that, while stellar masses are well determined, the SED fitting procedure does not strongly constrain star formation histories at high redshifts, where the uncertainties become larger due to the SFR-age-metallicity degeneracies. For this reason, the uncertainties associated with the SFR values estimated from the SED fitting are larger than those associated with the IR tracer.
In our analysis, we estimated star formation rates (along with stellar masses) using Bruzual \& Charlot (2003) synthetic models, fitting the whole 14 bands of photometry (from the $U$ band to $8 \mu \mathrm{m})$. We parameterize the star formation histories with a variety of exponentially declining laws (of timescales $\tau$ ranging from 0.1 to $15 \mathrm{Gyr}$ ), metallicities (from $Z=0.02 Z_{\odot}$ to $Z=2.5 Z_{\odot}$ ) and dust extinctions $(0<E(B-V)<1.1$, with a Calzetti or Small Magellanic extinction curve). Details are given in Table 1 of Fontana et al. (2004), in Fontana et al. (2006) and in Grazian et al. (2006, 2007). With respect to these recipes, the only difference in our method is the adoption of a minimum age of $0.1 \mathrm{Gyr}$. Below this value, the relation between UV luminosity and star formation rate changes rapidly with the age of the stellar population, leading to very high values of inferred SFRs. We are aware that exponential star formation histories may not be the correct choice in some cases. However, modeling in detail the star formation history of our galaxies is beyond the scope of the present paper, whose aim is to compare the star formation rates derived from the IR emission with those based on the widely used SED fitting procedures. In the following, we refer to this SFR estimation as $S F R_{\text {fit }}$.

We have also fitted our data using the Maraston (2005) (MR05) and Charlot \& Bruzual (in prep., see Bruzual 2007a,b) (CB07), including an improved TP-AGB stars treatment. The stellar mass estimates inferred using these new models are presented in Salimbeni et al. (2009) and are approximately 0.2 dex lower than those computed with Bruzual \& Charlot (2003) models. However, the extrapolated SFR values lead to a significant overestimate (especially in the case of Maraston (2005) models) of the SFR density that we present in Sect. 4.2. We suspect that part of this discrepancy is caused by the peculiar shapes in the near-IR side of the spectrum, which probably cause the worse $\chi^{2}$ than measured from the comparison with Bruzual \& Charlot (2003) models. Since a robust comparison among different stellar population models is beyond the scope of this paper, and pending further tests on the new MR05 and CB07 models, we continue to adopt the widely used Bruzual \& Charlot (2003) template library and refer to possible future work for more details on this point.

At $z>1.5$, it is possible to obtain an independent estimate of the SFR using the observed $L_{1500}$ and the observed slope of the UV continuum to estimate the $E(B-V)$, rather than the multiwavelength fit. In the following, we use the conversions adopted by Daddi et al. (2004), culling a sample of BzK-SF galaxies from our catalog and deriving the relevant SFR using the Daddi et al. (2004) scaling relations $S F R_{\mathrm{UV}} / M_{\odot} \mathrm{yr}^{-1}=L_{1500} / L_{0}$, with $L_{0}=8.85 \times 10^{27} \mathrm{erg} \mathrm{s}^{-1} \mathrm{~Hz}^{-1}$, and $A_{1500}=10 \times E(B-V)$, where $E(B-V)=0.25(B-z+0.1)_{A B}$. We refer to this SFR estimation as $S F R_{1500}$.

For clarity, SFRs lower than $0.01 M_{\odot} \mathrm{yr}^{-1}$ are assigned a value $0.01 M_{\odot} \mathrm{yr}^{-1}$ in the following analysis.

\subsection{Comparison between IR- and fit-based tracers}

We now discuss the consistency between the different SFR estimates. In this section, we use the $K_{\mathrm{s}}$-selected sample (subsample A) to ensure a proper sampling of the full SED, e.g., to ensure that all the bands, or most of them, are available for the fitting procedure.

We start from the redshift range $0.3-1.5$, plotting in Fig. 2 the comparison between $S F R_{\mathrm{IR}+\mathrm{UV}}$ and $S F R_{\mathrm{fit}}$. We include both the $24 \mu \mathrm{m}$ detected and undetected galaxies, for which only upper limits to $S F R_{\mathrm{IR}+\mathrm{UV}}$ can be obtained. These upper limits are misleading for the interpretation of the figure: since the SED fitting 

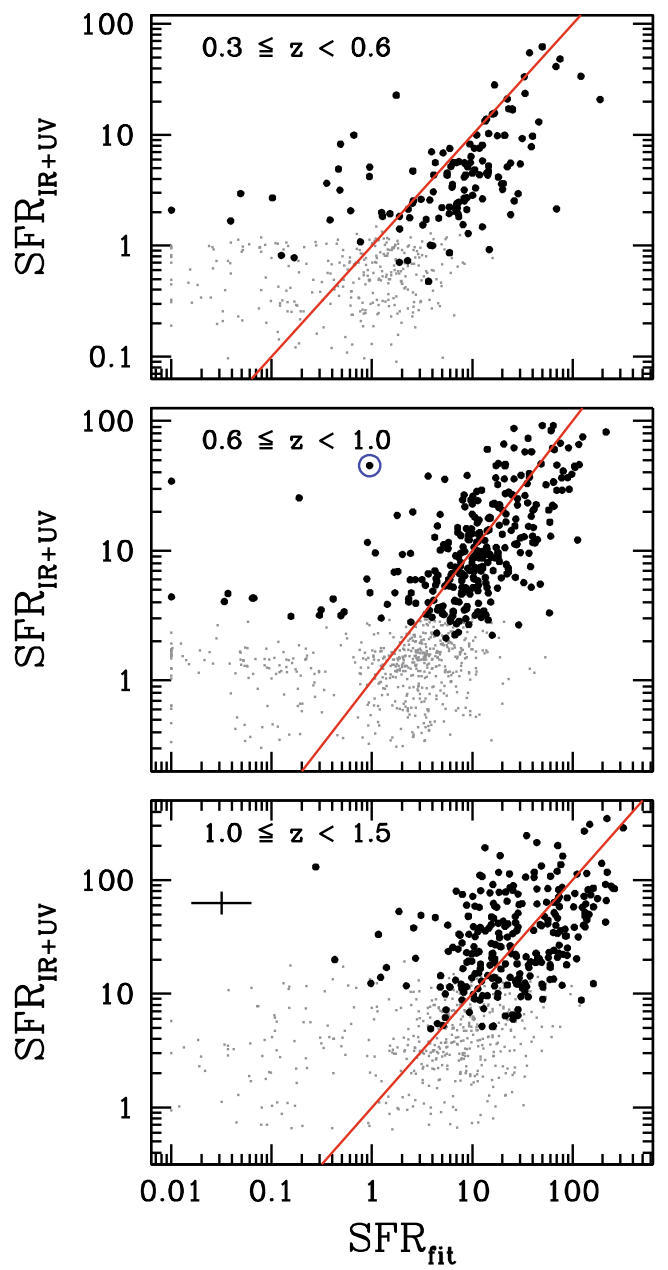

Fig. 2. Relation between $S F R_{\mathrm{IR}+\mathrm{UV}}$ and $S F R_{\mathrm{fit}}$ in three redshift bins for the whole $K_{\mathrm{s}}$-selected sample. Filled black dots are for the $F_{24 \mu \mathrm{m}}>$ $20 \mu \mathrm{Jy}$ subsample; small gray dots refer to galaxies undetected at $24 \mu \mathrm{m}$, and must be regarded as upper limits. The blue circle identifies an obscured AGN candidate, selected according to a similar technique as the one presented in Fiore et al. (2008) (see text). The red line defines the locus $S F R_{\mathrm{IR}+\mathrm{UV}}=S F R_{\mathrm{fit}}$. The typical uncertainties associated with both estimates are shown in the lower panel. The intrinsic parameter degeneracy involved with the SED fitting procedure is responsible for the larger uncertainties associated with $S F R_{\mathrm{fit}}$. Units are $M_{\odot} \mathrm{yr}^{-1}$.

can reach lower nominal values for the given SFR, the scatter in $S F R_{\mathrm{fit}}$ seems to be larger than that in $S F R_{\mathrm{IR}+\mathrm{UV}}$.

Given the many uncertainties involved in both estimators, the overall consistency between them appears reassuring. Apart from offsets and other systematics, which we discuss below, the majority of galaxies are assigned a consistent SFR, and the number of severe inconsistencies is small. These inconsistencies may have two different origins.

On the one hand, galaxies with $S F R_{\mathrm{fit}}$ much higher than $S F R_{\mathrm{IR}+\mathrm{UV}}$ can in principle be inferred from incorrect fitting of red galaxies. For these objects, the SED fitting could erroneously assign a large amount of dust to an otherwise dust-free, passively evolving population. Despite the relatively large number of passively evolving galaxies at $z<1.5$, the number of these misidentifications is very small at $z<1$, and low even at $z=1-1.5$.

On the other hand, galaxies with $S F R_{\mathrm{IR}+\mathrm{UV}} \gg S F R_{\mathrm{fit}}$ can be obtained either when the opposite misidentification occurs (e.g., for dusty star-forming galaxies fitted with a passively evolving SED) or, more interestingly, when the mid-IR emission is due to additional processes, not observable in the UV/optical regime.
Typical cases are AGN emission or additional star formation activity that is completely dust enshrouded. At $z<1.5$, these objects are again very rare in our sample. In particular, the IRbased SFR of the few galaxies with $S F R_{\mathrm{IR}+\mathrm{UV}} \simeq 2-6 M_{\odot} \mathrm{yr}^{-1}$ and $S F R_{\mathrm{fit}}<1 M_{\odot} \mathrm{yr}^{-1}$ at $z<1$ is probably overestimated, because of the incorrect application of a star-forming template to a more quiescent galaxy (see Fig. A.3).

For a more detailed discussion of the robustness of the fitbased SFR estimates, the related error analysis and $\chi^{2}$ contours, we refer the reader to Appendix B.

The existing systematic trends can be appreciated by examining Fig. 3a, where we plot the $S F R_{\mathrm{IR}+\mathrm{UV}} / S F R_{\mathrm{fit}}$ ratio for the $24 \mu \mathrm{m}$ detected sample. First, we note that the scatter in the $S F R_{\mathrm{IR}+\mathrm{UV}} / S F R_{\mathrm{fit}}$ distribution widens with redshift. This is probably due to a combination of effects: as we move to high redshifts, galaxies become intrinsically fainter and the rest-frame spectral coverage becomes narrower, making the SED fitting more uncertain; likewise, $24 \mu \mathrm{m}$ based indicators could become more uncertain as the filter moves away from the rest-frame mid-IR and approaches the PAH region.

More interestingly, a correlation between the $S F R_{\mathrm{IR}+\mathrm{UV}} / S F R_{\mathrm{fit}}$ ratio and $S F R_{\mathrm{IR}+\mathrm{UV}}$ can be observed. If we focus our attention on galaxies with star formation rates of between 10 and $100 M_{\odot} \mathrm{yr}^{-1}$, the $S F R_{\mathrm{IR}+\mathrm{UV}} / S F R_{\mathrm{fit}}$ distribution (black histograms) is centred on unity and fairly symmetric. In contrast, galaxies with milder activity (gray shaded histograms) have lower $S F R_{\mathrm{IR}+\mathrm{UV}} / S F R_{\mathrm{fit}}$ ratios, and we find a first hint of highly star-forming galaxies (red horizontally shaded histogram), i.e., objects with star formation rates higher than $100 M_{\odot} \mathrm{yr}^{-1}$, that have higher $S F R_{\mathrm{IR}+\mathrm{UV}} / S F R_{\mathrm{fit}}$ ratios.

The same systematic effects are seen at higher redshift. The upper panel of Fig. $3 \mathrm{~b}$ shows the ratio $S F R_{\mathrm{IR}+\mathrm{UV}} / S F R_{\mathrm{fit}}$ in the $1.5-2.5$ redshift range. It is immediately clear that the spread is larger than at lower redshifts, and that a large number of objects with $S F R_{\mathrm{IR}+\mathrm{UV}} / S F R_{\mathrm{fit}} \gg 1$ is observed. The observed spread is not surprising, since a similar disagreement, of up to two orders of magnitude in single galaxies at comparable redshifts, has already been noticed by the similar analysis of Papovich et al. (2006). At these redshifts, the faintness of the galaxies and the large $k$-corrections in the mid-IR are clearly even more effective in increasing the noise in both estimates. The factor of $\sim 2 \mathrm{shift}$ in the distribution of galaxies with SFRs of between 10 and $100 M_{\odot} \mathrm{yr}^{-1}$ is consistent with the uncertainty associated with the spectral library used to compute $L_{\mathrm{IR}}$ (see Appendix A).

It is therefore interesting to compare these estimates with the pure UV-based $S F R_{1500}$, described above. The relation between $S F R_{\mathrm{IR}+\mathrm{UV}}$ and $S F R_{\mathrm{IR}+\mathrm{UV}} / S F R_{1500}$ ratio is shown in the middle panel of Fig. 3b. Following Daddi et al. (2004), we applied this recipe only to the sample of BzK-SF in the redshift range of interest. The scatter is significantly lower than in the upper panel, but the same trend for exhibiting an IR excess still appears for highly star-forming objects.

To some extent, the excess in the mid-IR derived star formation rate is most probably caused by the presence of highly obscured AGNs. Daddi et al. (2007a) assumed that all the midIR excess objects drawn from the BzK-SF sample (i.e., all objects in the lower panel of Fig. 3b with $S F R_{\mathrm{IR}+\mathrm{UV}} / S F R_{1500}>3$ ) are powered by obscured AGNs. Following a different approach, Fiore et al. (2008) identified a population of highly obscured AGNs candidates in the entire $K_{\mathrm{s}}$-selected sample, selected on the basis of their very red spectrum $\left(F_{24 \mu \mathrm{m}} / F(R)>1000\right.$ and $R-K_{\mathrm{s}}>4.5$ ) (see also Dey et al. 2008). To use the fluxes directly measured in our catalog, we selected the very same objects following the criterium $F_{24 \mu \mathrm{m}} / F(I)>1000$ and $I-K_{\mathrm{s}}>4.5$, 

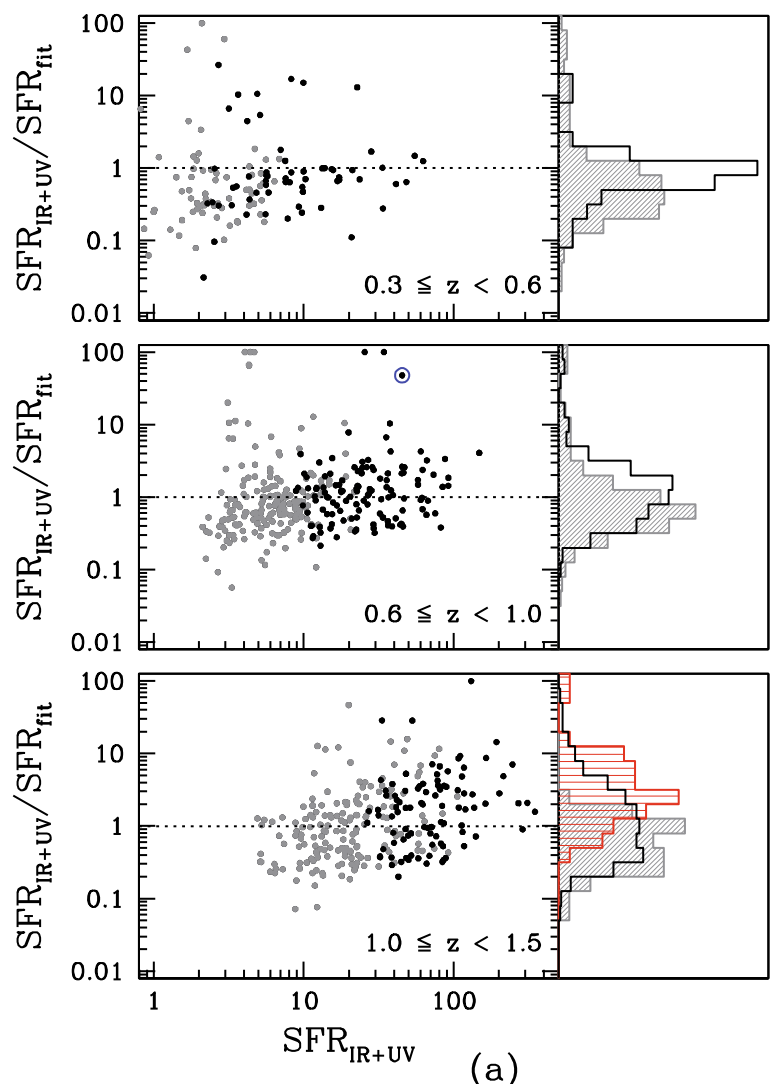

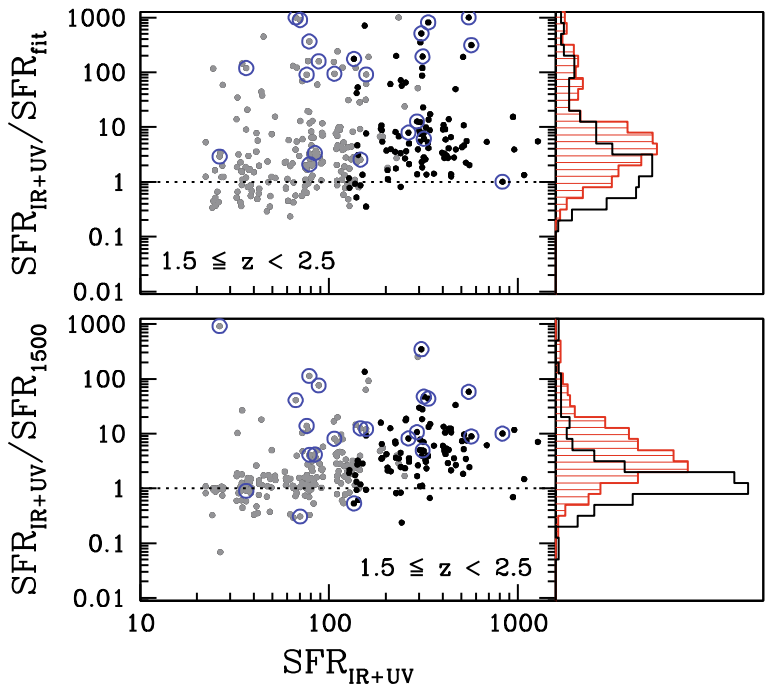

(b)

Fig. 3. Left panels in a) and left top panel in b): relation between $S F R_{\mathrm{IR}+\mathrm{UV}} / S F R_{\mathrm{fit}}$ ratio and $S F R_{\mathrm{IR}+\mathrm{UV}}$ in the different redshift bins for the $F_{24} \mu \mathrm{m}>$ $83 \mu \mathrm{Jy}$ (black dots) and $20<F_{24} \mu \mathrm{m}[\mu \mathrm{Jy}] \leq 83$ detected galaxies (gray dots); blue circles identify obscured AGN candidates, selected according to a similar technique as the one presented in Fiore et al. (2008) (see text). Left middle panel in b): relation between $S F R_{\mathrm{IR}+\mathrm{Uv}} / S F R_{1500}$ ratio and $S F R_{\mathrm{IR}+\mathrm{UV}}$ in the redshift bin 1.5-2.5 for the subsample of BzK-SF galaxies; symbols are as in the other panels. Right panels in a) and b): black plain, gray (diagonally) shaded and red (horizontally) shaded histograms show $S F R_{\mathrm{IR}+\mathrm{UV}} / S F R_{\mathrm{fit}}\left(S F R_{\mathrm{IR}+\mathrm{UV}} / S F R_{1500}\right.$ in the middle panel in b)) values respectively for $10<S F R_{\mathrm{IR}+\mathrm{UV}}\left[M_{\odot} \mathrm{yr}^{-1}\right]<100, S F R_{\mathrm{IR}+\mathrm{UV}}\left[M_{\odot} \mathrm{yr}^{-1}\right]<10$ and $S F R_{\mathrm{IR}+\mathrm{UV}}\left[M_{\odot} \mathrm{yr}^{-1}\right]>100$ samples. Units are $M_{\odot} \mathrm{yr}^{-1}$.

which we checked to be consistent with that used in Fiore et al. (2008). These objects are shown as blue open circles in Figs. 2 and 3 .

The presence of highly obscured AGNs could also produce the observed trend in the $S F R_{\mathrm{IR}+\mathrm{UV}} / S F R_{\mathrm{fit}}$ ratio, since they are expected to exist inside high mass galaxies, which are on average highly star-forming. A correlation between the fraction of obscured AGNs and the stellar mass was indeed shown in Daddi et al. (2007a).

However, we find that the trend in the $S F R_{\mathrm{IR}+\mathrm{UV}} / S F R_{\mathrm{fit}}$ ratio also extends to lower star formation rates (of average values lower than unity) and at lower redshifts. It is therefore possible that it reflects a change in the intrinsic physical properties of starforming galaxies. A possible explanation is related to metallicity effects. Galaxies with sub-solar metallicities have lower midIR emission (at least at $8 \mu \mathrm{m}$, Calzetti et al. 2007) and higher UV luminosity than solar ones, for a given level of SFR. The observed trend can therefore be related to a metallicity trend, which is natural to expect given the observed mass-metallicity relation from low to high redshifts (Maiolino et al. 2008, and references therein). Unfortunately, a direct check of the statement above is not feasible. Reliable metallicities cannot be inferred from broad-band SED fitting, and high resolution spectroscopy is necessary to properly distinguish between SEDs characterized by different lines and hence metallicities.

Alternatively, the observed trend can be taken as evidence of a failure in the assumption that a single attenuation law can adequately model the output from a star-forming galaxy.

\section{Mass assembly and downsizing}

We now discuss the star formation properties of our galaxy sample as a function of redshift and stellar mass. With respect to other surveys, our sample has the distinctive advantage of being selected by a multiwavelength approach. In this section, we use the subsample B. The $K_{\mathrm{s}}$ and $m_{4.5}$ cuts ensure a proper sampling of highly absorbed star-forming galaxies, and hence probably a complete census of all galaxies with high SFR. On the other hand, the deep $z$-selected sample contains the fainter and bluer galaxies of both low levels of dust extinction and low star formation rate.

In this catalog, we derive the SFR, which we refer to as IRbased, using the $S F R_{\mathrm{IR}+\mathrm{UV}}$ estimates to all objects with $F_{24 \mu \mathrm{m}} \geq$ $20 \mu \mathrm{Jy}$, and the $S F R_{\mathrm{fit}}$ to all fainter objects. We recall that the $S F R_{\mathrm{IR}+\mathrm{UV}}$ star formation rates are derived from the mid-IR emission, computed with the DH synthetic models, and by adopting the lowering correction of Papovich et al. (2007) for high SFRs. This technique was widely adopted in the literature, and we adopt it as a baseline. At the same time, we mention how the results would be changed by using $S F R_{\mathrm{fit}}$ for all the objects. We use the stellar mass estimates derived from the SED fitting analysis described above.

Finally, to avoid bias in the IR-based SFR estimates, we remove the obscured AGN candidates identified by Fiore et al. (2008) from our analysis. We are aware that the removal of this population will cause a reduction in the star formation rate measured on average at high masses. AGNs are indeed known 


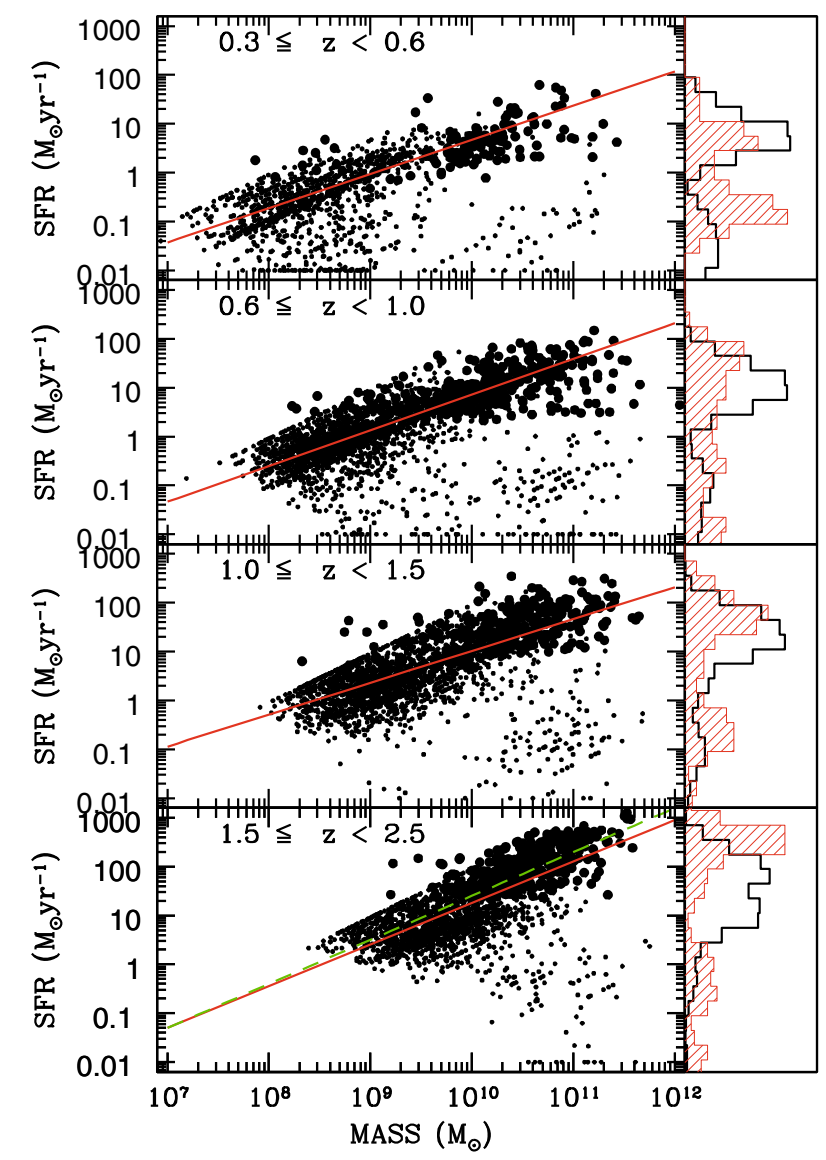

Fig. 4. Left panels: relation between the star formation rate and the stellar mass of the GOODS-MUSIC galaxies at different redshifts; large dots represent the galaxies with SFR derived from the $24 \mu \mathrm{m}$ emission, while small ones are $24 \mu \mathrm{m}$ undetected galaxies, with SFR derived from the SED fitting analysis; red solid lines show the $2 \sigma$-clipped least square fit described in the text; green dashed line in the highest redshift bin represents the correlation found by Daddi et al. (2007b). Right panels: SFR distribution in two mass bins, $10^{10}-10^{11} M_{\odot}$ (plain black histograms) and $10^{11}-10^{12} M_{\odot}$ (shaded red histograms).

to reside preferentially in high mass sources (Best et al. 2005; Daddi et al. 2007a; Brusa et al. 2009); although a high fraction of IR emission is understood to originate from accretion processes, some fraction of it probably originates from star formation.

\subsection{The stellar mass-SFR relation}

A direct relation between the stellar mass and the star formation rate in high redshift galaxies was identified both at $z \simeq 1$ (Elbaz et al. 2007) and in a subsample of star-forming BzK-SF at $z \simeq 2$ (Daddi et al. 2007b). To some extent, this disagrees with analogous trends for galaxy properties in the local Universe, where the most massive galaxies have very low levels of star formation rate (Heavens et al. 2004). We illustrate the global evolution in this relation by plotting in Fig. 4 the star formation rate of each galaxy as a function of its corresponding stellar mass.

It is immediately clear that a trend between star formation rate and stellar mass is present at all redshifts. This relation is of course neither tight nor unique: at all redshifts there exists a fraction of galaxies with low star formation rates, as also shown by the inset histograms. The SFR distribution becomes more and more bimodal with decreasing redshift. Figure 4 also shows that at the highest redshifts only the most massive galaxies are
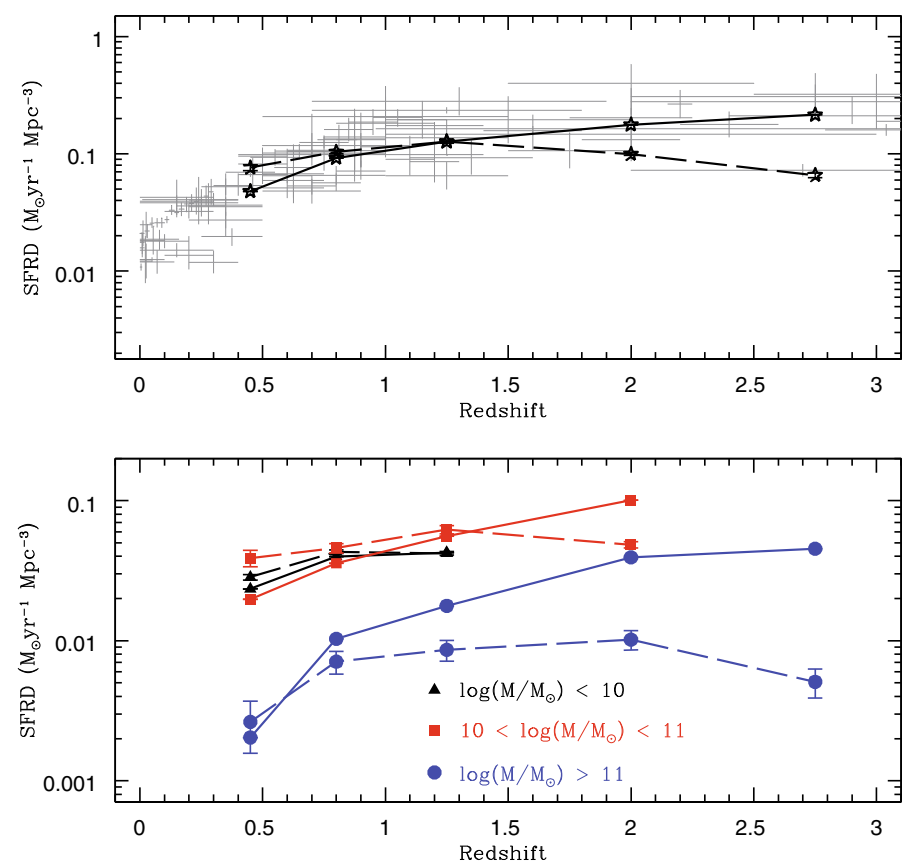

Fig. 5. Upper panel: the redshift evolution of the total cosmic SFRD. Solid lines refer to IR-based estimates, dashed lines to SED fitting estimates. The compilation of Hopkins \& Beacom (2006) is shown as small gray dots. Lower panel: the redshift evolution of the cosmic SFRD by galaxies of different masses. Only galaxies above the completeness limit in stellar mass at each redshift (see last column of Table 1) are shown. Solid lines refer to IR-based estimates, dashed lines to SED fitting estimates. Black, red and blue symbols correspond to different mass ranges as shown in the legend. Error bars include uncertainties on the SFR estimates and Poissonian errors.

already experiencing a quiescent phase. We note that we are incomplete at low masses and SFRs because of the magnitude limit of the sample. Nevertheless, there is a clear trend between SFR and $\mathrm{M}$ of the star-forming galaxies, and to quantify this, we computed a $2 \sigma$-clipped least squares fit, assuming the relation $S F R=A\left(M / 10^{11} M_{\odot}\right)^{\beta}$. We found values for $(A, \beta)$ equal to $(23.47,0.70),(38.93,0.73),(46.26,0.65),(127.98,0.85)$, respectively in the four redshift bins from $z \sim 0.3$ to $z \sim 2.5$. The increase in the normalization $A$ is robust, and caused by the global increase in the star formation rate with redshift. The steepening of the slope, instead, is less robust, since it depends on the threshold used for the $\sigma$-clipping and on the SED fitting parameters, e.g., the minimum galaxy age. With a $3 \sigma$-clipping, slopes can vary by $\sim 20 \%$. Considering these uncertainties, our results are in broad agreement with previous studies (Daddi et al. 2007b; Elbaz et al. 2007).

It is interesting to note that the correlation between SFR and stellar mass holds also using the $S F R_{\text {fit }}$ estimates. While the two correlations are consistent at intermediate redshifts, the slope of the $S F R_{\text {fit }}$ one is steeper at low redshift (0.90) and milder at high redshift (0.42), because of the systematic trends shown in Fig. 3.

\subsection{The evolution in the cosmic star formation rate density}

The most concise representation of the evolution in the star formation rate across cosmic time is the star formation rate density (SFRD), which we show in Fig. 5. We show both the IR-derived estimates (continuous lines) as well as those obtained purely by the SED fit (dashed line). In this figure only, we extend the analysis to the redshift bin $2.5<z<3$. In this redshift regime, 
the IR templates are hardly representative, since the $24 \mu \mathrm{m}$ band is no longer representative of the dust emission peak, although the Dale \& Helou (2002) models are still formally applicable. For these reasons, the $S F R_{\mathrm{IR}+\mathrm{UV}}$ estimate is tentative and should be interpreted with caution.

Because of the complex selection criteria that we adopted, it was not easy to compute the corrections necessary to include the contribution of galaxies fainter than those included in our sample. Because of our mid-IR selection, we assumed that we include nearly all highly star-forming galaxies. The missed fraction consists therefore mainly of galaxies with low star formation activity, sampled by the $z$-selection. A good approximation of our incompleteness can be obtained by fitting the star formation rate function computed on the $z$-selected sample in each redshift bin. Volumes were computed with the $1 / V_{\max }$ method. We found that the SFR functions can be fitted by Schechter functions of slopes $\sim-1.5$. We then corrected our observations for these contributions at low SFR values by extrapolating from the fitted functions. The corrections are small for $z<2.5$, of the order of $10 \%$, consistent with the assumption that the sample is complete at high star formation rate values. At $z>2.5$, the corrections are higher than $50 \%$.

We first note that the total SFRD (upper panel of Fig. 5) which we derived from our sample (black stars) reproduces well the compilation of other surveys completed by Hopkins \& Beacom (2006), normalized to a standard Salpeter (1955) IMF, and is also in agreement with Le Floc'h et al. (2005), Caputi et al. (2007) and Rodighiero et al. (2009).

It is more interesting to study the trend in the SFRD for galaxies of different stellar masses (lower panel of Fig. 5). Previous surveys, such as Combo-17 (Zheng et al. 2007, that traced the evolution in the SFRD to $z \simeq 1$ ) and the GDDS one (Juneau et al. 2005, which first presented the evolution in the SFRD in a $K_{\mathrm{s}}$-selected sample to $z \simeq 2$ ), demonstrated that the contribution to the SFRD by the more massive galaxies (typically those with $M>10^{11} M_{\odot}$ ) is negligible at low redshift, and becomes much larger at $z \gg 1$ corresponding to the major epoch of formation in the more massive galaxies. Our data confirm this picture, with an increase by a factor 20 from $z \simeq 0.5$ to $z \simeq 2$. The sharp decline at low $z$ suggests that these structures must have formed their stars at earlier epochs. Keeping in mind the caveat mentioned above about the reliability of $S F R_{\mathrm{IR}+\mathrm{UV}}$ at $z>2.5$, we note that the increase in the star formation rate density in $M>10^{11} M_{\odot}$ galaxies appears to halt at $z>2.5$. This is expected since, at these $z$, the number density of $M>10^{11} M_{\odot}$ galaxies drops quickly (Fontana et al. 2006; Marchesini et al. 2008).

The evolution in the SFRD for galaxies of different stellar masses is one of many pieces of evidence of downsizing. Indeed, downsizing is evident as an evolution in the slope of the SFRD as a function of redshift, which becomes yet steeper as high mass galaxies are considered because of the more rapid evolution of massive galaxies, wherever a number of physical processes are expected to suppress the star formation more efficiently than in lower mass galaxies.

The exact values of the SFRD depend very sensitively on the mass ranges adopted. Our mass bin choice is determined by the intention of having good statistics in each bin. Keeping in mind this warning, our observations seem to support the downsizing picture for the reasons mentioned above. Indeed, in the redshift interval 0.3-1.5, where all mass samples are complete, the SFRD derived from the IR emission increases by a factor 1.8, 2.75, and 9 , respectively for bins of increasing mass. We also detect a similar steepening in the slope of the relation between the average star formation rate per unit mass (which is treated in Sect. 4.3) and redshift between low and high mass galaxies.

At all observed redshifts, the more massive galaxies do not dominate the global SFRD. To some extent, this may follow from the exact choice of the mass bins. However, it is easy to predict that the major contributors to the global SFRD are galaxies immediately below the characteristic mass $M^{*}$. The number of galaxies of given mass $N(M)$ at any redshift can be represented as a Schechter function $\left(N(M) \propto\left(M / M^{*}\right)^{\alpha} \exp \left(-M / M^{*}\right)\right)$. Given the tight correlation between stellar mass and star formation rate described above $\left(\dot{M} \propto M^{\beta}\right)$, and assuming that the fraction of star-forming galaxies is $f(M)$, the contribution to the cosmic SFRD for galaxies in a logarithmic interval $d \log M$ is simply

$$
\frac{\mathrm{d}(S F R D(M))}{\mathrm{d} \log (M)} \propto\left(M / M^{*}\right)^{\alpha+\beta+1} \exp \left(-M / M^{*}\right) f(M) .
$$

Since $\alpha$ is in the range $\simeq-1.2 /-1.4$ (Fontana et al. 2006) and $\beta \simeq 0.6-0.9$ (see above, relating to the IR-based estimate), and ignoring for the moment $f(M)$, the shape of the $\mathrm{d}(\operatorname{SFR} D(M)) / \mathrm{d} \log (M)$ is that of a Schechter function with positive slope, which has a peak around $M^{*}$ and decreases at $\log (M)<\log \left(M^{*}\right)$. The shape of $f(M)$ is somewhat more uncertain. We simply assume that the fraction of active galaxies above the characteristic mass is around 0.5 at $z \simeq 1$, around 0.8 at $z \simeq 2$, and will probably be higher at lower masses (around 0.8 at $z \simeq 1$ and around unity at $z \simeq 2$ ). As a result, it further decreases the distribution of $\mathrm{d}(\operatorname{SFR} D(M)) / \mathrm{d} \log (M)$ at high masses, without affecting the low mass regime.

Using the parameters of the galaxy mass function obtained in Fontana et al. (2006), and the SFR-stellar mass correlation shown above, we computed the expected $\mathrm{d}(\operatorname{SFRD}(M)) / \mathrm{d} \log (M)$ distribution in two redshift bins, $0.8-1.2$ and 1.5-2, where data from different surveys (Zheng et al. 2007; Juneau et al. 2005) could be combined. To convert masses and SFRs of Zheng et al. (2007) from a Chabrier (2003) to a Salpeter (1955) IMF, we used a factor of 1.78 (Bundy et al. 2006) and 1.5 (Ferreras et al. 2005), respectively. Masses and SFRs of Juneau et al. (2005) were instead renormalized from a Baldry \& Glazebrook (2003) to a Salpeter (1955) IMF using the factors 1.82 (Juneau et al. 2005) and 2 (Hopkins \& Beacom 2006). We note that the argument presented above is unaffected by the well known discrepancy between the directly measured mass density and the one inferred from the integration of the star formation rate density (Wilkins et al. 2008). Our point here does not concern the integrated amount of stars formed, but only the analytical shape of the SFRD.

The result is shown in Fig. 6, where we plot the expected $\mathrm{d}(\operatorname{SFR} D(M)) / \mathrm{d} \log (M)$ distribution by assuming both $f(M)=1$ and a $f(M)$ that decreases with both increasing mass and decreasing redshift. Our data are in good agreement with those provided by Zheng et al. (2007), who inferred SFR estimates using the same method that we adopt to compute $S F R_{\mathrm{IR}+\mathrm{UV}}$. In contrast, Juneau et al. (2005) estimated the SFR from the rest-frame UV continuum, and obtained values lower than $S F R_{\mathrm{IR}+\mathrm{UV}}$. The differences can probably be attributed to the different selection criteria and SFR estimators used in their work.

This analysis confirms that the major contribution to the star formation is from galaxies of masses around or immediately below the characteristic mass $M^{*}$. This statement is consistent with the evolution in the SFRD for different mass bins that we show in Fig. 5. The combination of the steepening in the SFR-M relation, the increase in the SFR values with increasing redshift, and the lower fraction of active galaxies at low $z$, is more significant 

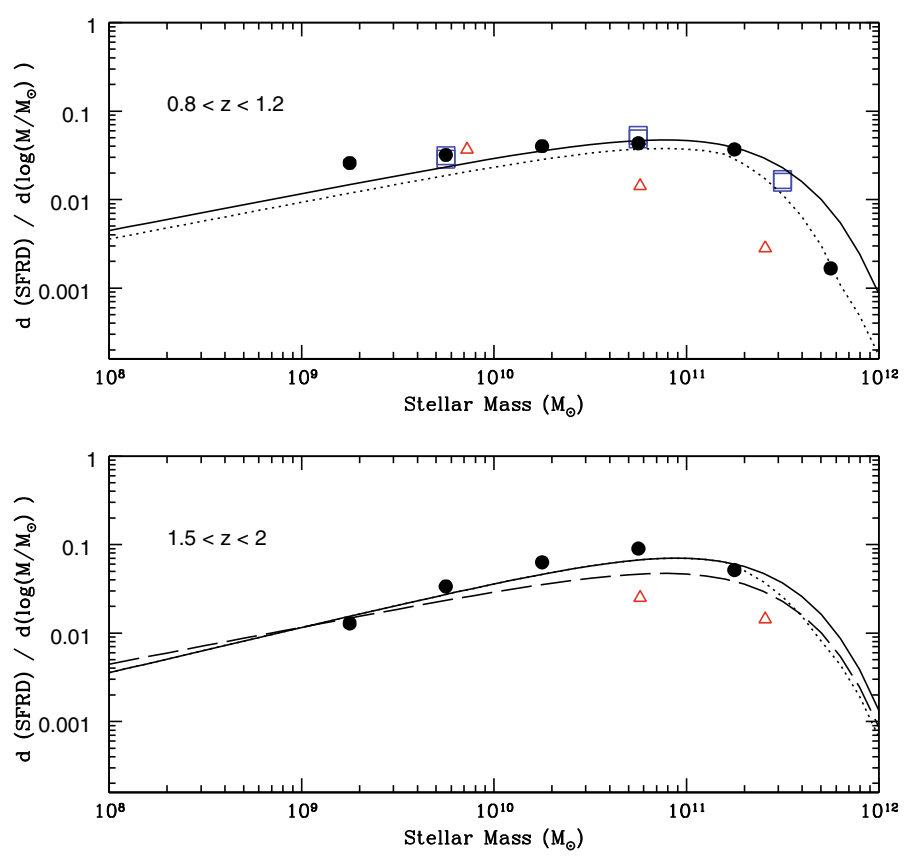

Fig. 6. The contribution to the cosmic SFRD by galaxies of given mass, in two redshift bins. Solid and dashed lines are computed assuming $f(M)=1$. The dashed line in the lower panel correspond to the $z \simeq 1$ line of the upper panel. Dotted lines are computed assuming $f(M) \neq 1$ (see text). Black filled dots: this work; blue squares: Combo-17 (Zheng et al. 2007); red open triangles: GDDS (Juneau et al. 2005).

than the effect of the decrease in the characteristic stellar mass at increasing $z$.

\subsection{The specific star formation rate and the comparison with theoretical models}

In Fig. 7, we plot the relation between the stellar mass and the specific star formation rate (SSFR hereafter) for all galaxies divided into redshift bins. To be able to compare our findings with the Millennium Simulation predictions, we converted our masses and SFR to the Chabrier (2003) IMF used by the Millennium Simulation.

We recall that our SSFR estimates are quite low compared to the possible estimates made by adopting standard values for $S F R_{\mathrm{IR}+\mathrm{UV}}$. On the one hand, the conversion between mid-IR flux and SFR includes a lowering factor for high SFRs, as described in Sect. 3.1 and in Appendix A. On the other side, the use of more recent models of stellar populations (Maraston 2005) would slightly decrease the average stellar masses (by $20 \%$ on average, see Salimbeni et al. 2009), and hence increase the derived SSFR.

First of all, we notice a strong bimodality in the SSFR distribution. Two distinct populations, together with some sources lying between the two, are detectable, one of young, active and blue galaxies (the so-called blue cloud) and the other one consisting of old, "red and dead", early-type galaxies (red sequence) (see also Fig. 4). The loci of these two populations are consistent with the selection in Salimbeni et al. (2008) between early- and late-type galaxies. It is noticeable how these evolved objects are already in place even at the highest redshift. We reserve a more complete discussion of these objects to Fontana et al. (2009).

A trend for the specific star formation rate to increase with redshift at a given stellar mass is evident: galaxies tend to form
Table 1. Average observed IR- and fit-based SSFR at different redshifts for galaxies above the completeness limit in stellar mass.

\begin{tabular}{cccc}
\hline \hline \multicolumn{4}{c}{$\left\langle\right.$ SSFR $\left.\left[\mathrm{Gyr}^{-1}\right]\right\rangle$} \\
\hline$\Delta z$ & IR+UV & SED fitting & $M_{\lim }\left[M_{\odot}\right]$ \\
\hline $0.3-0.6$ & $0.278 \pm 0.022$ & $0.570 \pm 0.137$ & $5 \times 10^{9}$ \\
$0.6-1.0$ & $0.487 \pm 0.017$ & $0.658 \pm 0.103$ & $8 \times 10^{9}$ \\
$1.0-1.5$ & $0.754 \pm 0.029$ & $0.854 \pm 0.168$ & $2 \times 10^{10}$ \\
$1.5-2.5$ & $1.652 \pm 0.058$ & $0.420 \pm 0.122$ & $7 \times 10^{10}$ \\
\hline
\end{tabular}

their stars more actively at higher redshifts. The bulk of active sources shifts to higher values of SSFR with increasing redshift. Our findings are in good agreement with Pérez-González et al. (2005) and Papovich et al. (2006).

A significant fraction of the sample, increasing with redshift, is in an active phase. It is natural to compare the SSFR (which has units of the inverse of a timescale) with the inverse of the age of the Universe at the corresponding redshift $t_{U}(z)$. We define galaxies with $M / S F R<t_{U}(z)$ as "active" in the following, since they are experiencing a major episode of star formation, potentially building up a substantial fraction of their stellar mass in this episode ${ }^{3}$. Galaxies selected following this criterium are forming stars more actively than in their recent past.

At $1.5 \leq z<2.5$, the fraction of active galaxies in the total sample is $66 \%$, and their mean SFR is $309 M_{\odot} \mathrm{yr}^{-1}$. To compute the total stellar mass produced within this redshift interval, it is necessary to know the duration of the active phase. For this purpose, we use a duty cycle argument and suppose that the active fraction of galaxies is indicative of the time interval spent in an active phase. We adopt the assumption that the active fraction is stable within the redshift bin considered. The time spanned in the 1.5-2.5 redshift interval corresponds to 1.5 Gyr. By multiplying the fraction of active galaxies by the time available, we derived an average duration of the active phase of 0.99 Gyr. The average amount of stellar mass assembled within each galaxy during these bursts is measured to be the product of the average SFR and the average duration of the active phase, and equals to $3.1 \times 10^{11} M_{\odot}$, representing a significant fraction of the final stellar mass of the galaxies considered. Although quite simplified, this analysis implies that most of the stellar mass of massive galaxies is assembled during a long-lasting active phase at $1.5 \leq z<2.5$. It is important to remark that this process of intense star formation occurs directly within already massive galaxies, and, given its intensity, prevails throughout growth episodes due to merging events of already formed progenitors. A similar point is also stated by Daddi et al. (2007b). Independent arguments converging on the same result are based on the tightness of the SFR-mass relation (Noeske et al. 2007), on the kinematics of disks (Cresci et al. 2009) and on the analysis of the accretion histories of dark matter haloes in the Millennium Simulation (Genel et al. 2008).

To provide a further physical insight in this process, we compared our results with the predictions of three recent theoretical models of galaxy formation and evolution. Our sample is affected by mass incompleteness, so only galaxies above the completeness limit in each redshift bin were considered in the comparison. We note that this limit depends on the redshift bin. In Table 1, we report the average SSFR as a function of the redshift bin for the IR- and the fit-based estimates, along with the

\footnotetext{
3 Indeed, if $M=\langle S F R\rangle_{\text {past }} \times t_{U}(z)$, where $\langle S F R\rangle_{\text {past }}$ is the star formation rate averaged over the whole age of the Universe at the corresponding $z$, the requirement $S F R / M>\left(t_{U}(z)\right)^{-1}$ implies $S F R>\langle S F R\rangle_{\text {past }}$.
} 


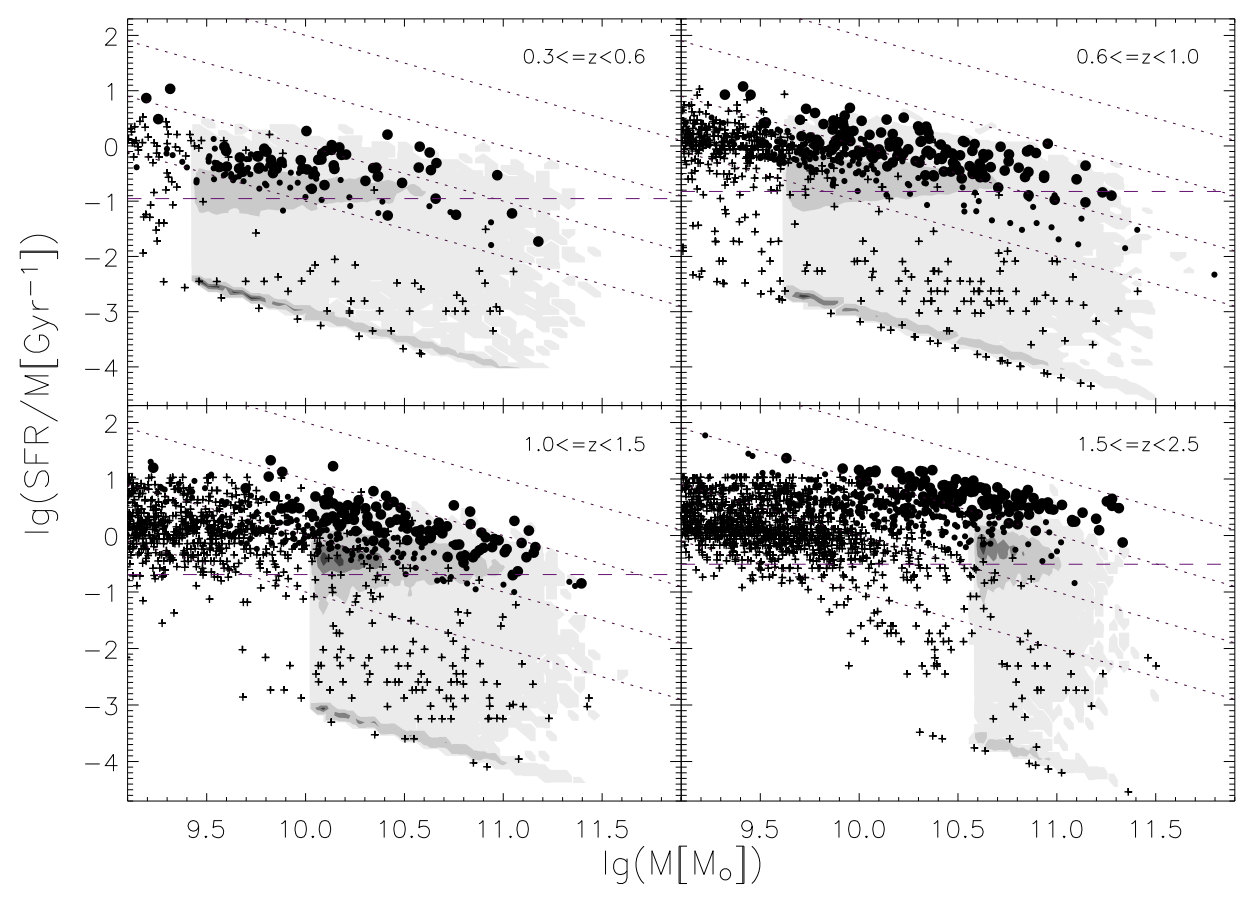

Fig. 7. Relation between the specific star formation rate and the stellar mass calibrated to a Chabrier (2003) IMF. Larger dots correspond to $24 \mu \mathrm{m}$ sources with $F_{24} \mu \mathrm{m}>83 \mu \mathrm{Jy}$, while smaller ones show $24 \mu \mathrm{m}$ detections with $20 \mu \mathrm{Jy}<F_{24 \mu \mathrm{m}} \leq 83 \mu \mathrm{Jy}$. Plus refer to $24 \mu \mathrm{m}$ upper limits. Dotted lines correspond to constant SFRs of 1, 10, 100 and $1000 M_{\odot} \mathrm{yr}^{-1}$. The horizontal dashed lines indicate the inverse of the age of the Universe at the centre of each redshift interval. Shaded contour levels (at $0.05 \%, 10 \%, 50 \%$ and $80 \%$ level) represent the predictions of the Kitzbichler \& White (2007) rendition of the Millennium Simulation.

completeness mass cuts computed as described in Fontana et al. (2006). SSFRs and masses are calibrated to a Salpeter (1955) IMF.

In Fig. 7, we show the predictions of the semi-analytical rendition of Kitzbichler \& White (2007) of the Millennium $N$-body dark matter Simulation (Springel et al. 2005; Lemson \& Springel 2006; De Lucia \& Blaizot 2007), which adopts a WMAP1 cosmology. We find that the model predicts an overall trend that is consistent with our findings. The SSFR decreases with stellar mass (at given redshift) and increases with redshift (at given stellar mass). In addition, it forecasts the existence of quiescent galaxies even at $z>1.5$. However, the average observed SSFR is systematically under-predicted (at least above our mass limit) by a factor $\sim 3-5$ by the Millennium Simulation. A similar trend for the Millennium Simulation at $z \sim 2$ was already shown by Daddi et al. (2007b).

We also compared our findings with the semi-analytical models of Menci et al. (2006) (adopting a cosmology consistent with WMAP1) and MORGANA (Monaco et al. 2007, updated by Lo Faro et al. 2009, adopting a WMAP3 cosmology). They show very similar trends with respect to the Millennium Simulation, with only slightly different normalizations.

In Fig. 8, we plot the average SSFR as a function of the redshift bin for the IR- and the fit-based estimates as well as those predicted by the three theoretical models. Once again, we only consider galaxies above the completeness limit in mass for each redshift bin (see last column in Table 1). The trend depicted must therefore not be considered an intrinsic trend, since we observe different populations at the different redshifts because of the mass cuts. Errors in the average SSFR were estimated by a Monte Carlo simulation.

We find that although all models taken into account reproduce the global observed trend, they predict an average star
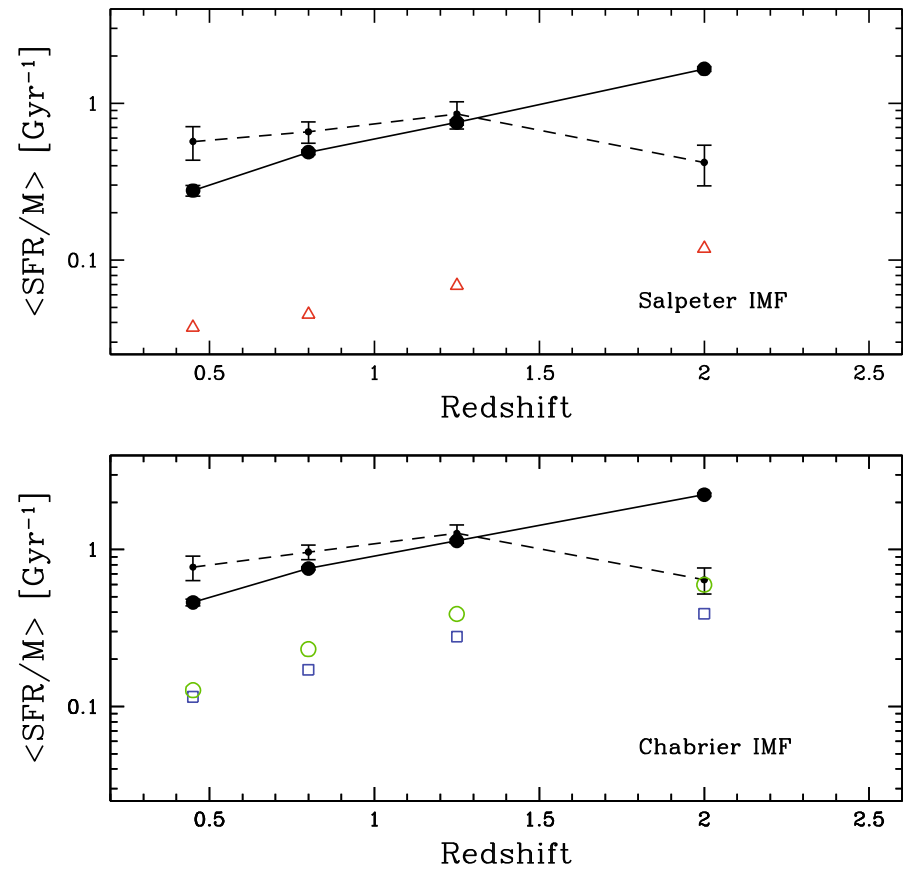

Fig. 8. Average SSFR in each redshift bin. Black solid lines refer to IR-based estimates, dashed lines to the SED fitting estimate. Error bars for the fit-based estimate are larger than those of the IR-based one because of the intrinsic parameter degeneracy involved with the SED fitting procedure. In the upper panel, we show the comparison with Menci et al. (2006) semi-analytical model (red triangles), which adopts a Salpeter (1955) IMF. In the lower panel we compare our data with the predictions of the Millennium Simulation (Kitzbichler \& White 2007) (blue squares) and MORGANA (green circles), which adopt a Chabrier (2003) IMF. The comparison is done above the completeness limit in stellar mass, which is different in each redshift bin (see text and Table 1). 


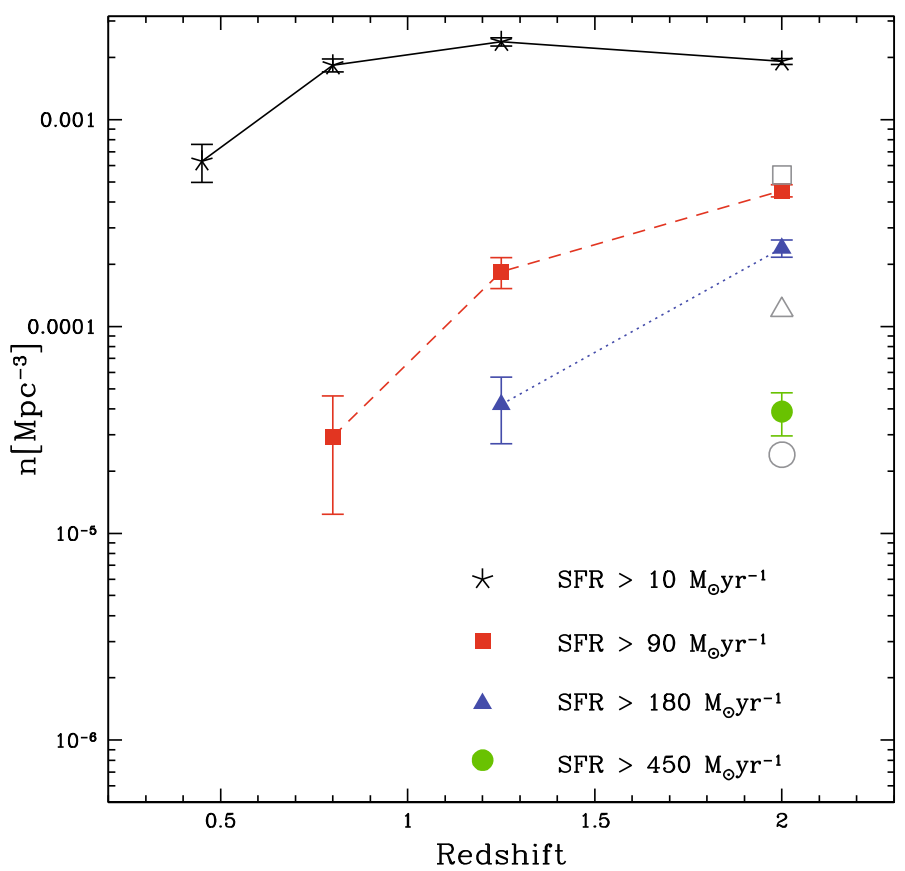

Fig. 9. Number density of star-forming galaxies as a function of redshift and lower SFR. Filled symbols represent our observations corresponding to the SFR thresholds shown in the legend. Open gray symbols at $z \sim 2$ are the predictions of Khochfar \& Silk (2008) model.

formation activity that is lower than observed in most of the mass regimes. The observed star formation occurring in situ inside massive galaxies is higher than predicted by a factor 3-5 for the Millennium Simulation and for MORGANA, and around 10 for Menci et al. (2006) model. Daddi et al. (2007b) claimed that the star formation rates predicted by the Millennium Simulation are as much as one order of magnitude lower than those observed at $z \sim 2$. Similar mismatch at these redshifts between observed SFRs and those expected by various kind of theoretical models, independent of their physical process implementations, were found and discussed by Davé (2008). To reconcile data and model predictions, Khochfar \& Ostriker (2008) proposed a galaxy formation scenario at high redshift that is mainly driven by cold accretion flows. Their model allows an increased star formation efficiency resulting in a closer agreement with observations.

We then considered the number density of galaxies with star formation rates higher than a fixed threshold as a function of redshift, and compared our observations with the model of Khochfar \& Silk (2008) at $z \sim 2$. Figure 9 compares our data (filled symbols) with the theoretical predictions (open symbols). The SFR limits were chosen to allow comparison with Khochfar $\&$ Silk (2008) results ${ }^{4}$. The agreement is very good for objects with $S F R>90 M_{\odot} \mathrm{yr}^{-1}$, while the model slightly underpredicts the number density of galaxies with higher levels of star formation rate.

A more comprehensive comparison between theoretical predictions and observations was presented in Fontanot et al. (2009).

\footnotetext{
${ }^{4}$ Note that they adopt a Chabrier (2003) IMF, so the SFR thresholds have been renormalized by adopting the conversion factors in Sect. 4.2.
}

\section{Summary and discussion}

We have presented a revised version of our GOODS-MUSIC photometric catalog for the GOODS-S field. The major new feature of this release, on which our scientific discussion is based, is the inclusion of $24 \mu \mathrm{m}$ data taken from the Spitzer MIPS public images, of which we provide a self-consistent photometry for each object in the catalog. We employed a PSF-matching technique, performed by ConvPhot software (De Santis et al. 2007), to measure $24 \mu \mathrm{m}$ photometry by exploiting the high resolution $z$-ACS image used as a prior to detect the objects' positions. This allows us to reduce the effects of confusion noise and deblending problems caused by the large size of the MIPS PSF.

We have used this new catalog to study the star-forming properties of galaxies up to $z \simeq 2.5$. We have first compared the estimates of the SFR obtained from the total IR luminosity $\left(S F R_{\mathrm{IR}+\mathrm{UV}}\right)$ and from the SED fitting analysis to the overall 0.3 to $8 \mu \mathrm{m}$ photometry $\left(S F R_{\text {fit }}\right)$, which are the two major estimators of the SFR that have been used so far in high redshift galaxies. We found that the two tracers are consistent overall, especially in the redshift range $0.3-1.5$. The overall median ratio of $S F R_{\mathrm{IR}+\mathrm{UV}}$ to $S F R_{\mathrm{fit}}$ is around unity, a limited number of objects appearing to have discrepant results. The agreement between the two estimators appears to depend on redshift because the scatter increases with redshift. Moreover, $S F R_{\mathrm{fit}}$ is slightly systematically overestimated with respect to $S F R_{\mathrm{IR}+\mathrm{UV}}$ in the lowest redshift bin (0.3-0.6). The scatter increases significantly at $z>1.5$, where the IR+UV value is systematically larger than the one resulting from the SED fitting analysis for bright objects. However, these results stem from a systematic trend that holds at all redshifts. In galaxies with star formation rates $<10 M_{\odot} / y r$, the fit-derived SFR is on average higher than $S F R_{\mathrm{IR}+\mathrm{UV}}$, while the opposite holds at $S F R>100 M_{\odot} / y$ r. It is at present difficult to ascertain the origin of this systematic trend. It can be due to systematics that affect the interpretation of the data, either from the SED fitting technique as the target galaxies move in redshift, or in the templates used in the extrapolation of the mid-IR observed flux, especially at high redshift, where it samples the PAH region. Alternatively, it could have physical origins, such as a metallicity trend or a failure in the assumption that a single attenuation law can adequately model the output from a star-forming galaxy.

Keeping in mind these uncertainties in the estimate of the star formation rate, we summarize the basic results obtained by adopting $S F R_{\mathrm{IR}+\mathrm{UV}}$, which we assume to be a more reliable tracer because it is not affected by dust extinction.

- We show that, at all redshifts considered here, there is a correlation between the stellar mass and the star formation rate of star-forming galaxies. The logarithmic slope of this correlation, after applying a $\sigma$-clipping algorithm to remove all quiescent galaxies, is in the range $0.6-0.9$, with some indications of a steepening with increasing redshift.

- The SFRD derived from our sample agrees with the global trend already depicted by other surveys. When divided according to stellar mass, it shows that more massive galaxies enter their active phase at redshifts higher than lower mass ones. At $z>2.5$, the increase in the SFRD due to the more massive galaxies (with $M>10^{11} M_{\odot}$ ) appears to halt, in broad agreement with the expectations of theoretical models (Menci et al. 2004; Bower et al. 2006). This is mainly because galaxies of higher mass become extremely rare at these redshifts. The increase in the slope of the SFRD with redshift of samples of increasing mass seems to support the downsizing scenario. 
- At all redshifts, the main contributors to the cosmic SFRD are galaxies around, or slightly below, the characteristic stellar mass $M^{*}$.

- Massive galaxies at $z \simeq 2$ are vigorously forming stars, typically at a rate of $300 M_{\odot} \mathrm{yr}^{-1}$. A simple duty-cycle argument (see Sect. 4.3) suggests that they assemble a significant fraction of the final stellar mass during this phase.

- The specific star formation rate of our sample shows a welldefined bimodal distribution, with a clear separation between actively star-forming and passively evolving galaxies.

While these results are grossly independent of the particular star formation rate estimate, the specific details appear to depend on the chosen indicator. In particular, the correlation between the SFR and the stellar mass still holds using $S F R_{\text {fit }}$, but it is steeper at $z \simeq 0.5$ and it becomes flatter at high redshift, remaining similar in the two intermediate redshift bins. As far as the star formation rate density and the specific star formation rate are concerned, the two techniques used to estimate the SFR provide consistent results up to $z \sim 1$.5. However, significant differences are evident at redshift $\gtrsim 1.5$, where the IR-based SFRD flattens and the fit-based one begins to decline. The average IR-based SSFR increases monotonically to the highest observed redshifts, while the fit-based one has a turn-over around $z \sim 1.5$ and then decreases. The trends above directly reflect the correlation between $S F R_{\mathrm{IR}+\mathrm{UV}}$ and $S F R_{\mathrm{fit}}$.

We used our results for the redshift evolution in the specific star formation rate, and its trend with the stellar mass, to investigate the predictions of a set of theoretical models of galaxy formation in $\Lambda$-CDM scenario.

On the one hand, these models reproduce the global trend that we find in the data - the most important being the increase in the specific star formation rate with redshift, and its trend with stellar mass. Somewhat surprisingly, however, the average SSFR of galaxies in our sample is significantly higher than predicted by theoretical models, in most of the mass regimes. Essentially, after including a strict completeness limit in stellar mass, we found that the typical SSFR of galaxies at a given mass is a factor at least $\simeq 3-5$ higher than predicted by the models we have considered here. This mismatch is very clear for massive galaxies $\left(M_{*} \simeq 10^{11} M_{\odot}\right)$ at $z \simeq 2$ and for less massive galaxies at $z \simeq 1$, where we are able to complete a higher quality statistical analysis.

It is not obvious to ascertain the origin of this mismatch. It could be caused by a genuine failure of the models. These models often quench the star formation in massive galaxies to prevent the formation of blue, giant galaxies at low redshift, and to reproduce the existence of red, massive galaxies at high redshift. The mismatch that we observe could imply that the feedback and star formation recipes adopted are too simplified or incorrect. Most of the models considered in this work adopt the commonly used star formation scenario, where gas uniformly collapsing towards the galaxy centre forms a stable disk. New processes are being explored (e.g., Dekel et al. 2009) that involve a more rapid formation of galaxies by cold streams and could lead to closer agreement with observations.

Alternatively, the mismatch could be related to the overestimate of the stellar mass of the typical star-forming galaxy - for instance, due to a combination of the overestimate of merging events and of the star formation activity in its past history, i.e., at redshifts higher than those sampled in this work. A possible consequence of this is the overestimate of the stellar mass function predicted by the Millennium Simulation at high redshifts in relation to observations (Kitzbichler \& White 2007).
Additional evidence of an incorrect treatment of the star formation processes was presented by Fontanot et al. (2009) and Lo Faro et al. (2009). In their models, these authors found an excess of low mass galaxies at $z<2$ and faint LBGs at $z>3$, respectively, which is probably counterbalanced by the suppression of the star formation to reproduce the observed evolution in the SFRD.

However, we remark that the interpretation of the observations is affected by a number of uncertainties, such as uncertainties in the stellar mass estimates or uncertainties originating from both the templates used to convert $24 \mu \mathrm{m}$ fluxes into total infrared luminosities (see Appendix A) and the SED fitting analysis. An example of these uncertainties is illustrated by the mismatch between the integrated star formation rate density and the stellar mass density. This disagreement could be alleviated by an evolving IMF (Wilkins et al. 2008; Davé 2008), which would provide lower values of SFR.

To make conclusive, quantitative statements in this direction, we ultimately need to improve the reliability of the SFR measurements, especially for high redshift galaxies. Forthcoming IR facilities, such as Herschel and ALMA, will probably provide us with a more coherent picture.

Acknowledgements. We are grateful to Pierluigi Monaco, Niv Drory and Andrew Hopkins for the useful discussions. Observations were carried out with the Very Large Telescope at the ESO Paranal Observatory under Program IDs LP168.A-0485 and ID 170.A-0788 and the ESO Science Archive under Program IDs 64.O-0643, 66.A-0572, 68.A-0544, 164.O-0561, 163.N-0210, and 60.A9120. The Millennium Simulation databases used in this paper and the web application providing online access to them were constructed as part of the activities of the German Astrophysical Virtual Observatory. This work has been partly funded by ASI, under the COFIS contract.

\section{Appendix A: The estimate of total infrared luminosity}

We describe our method to convert mid-IR fluxes into total infrared luminosities and compare the results obtained from different template libraries.

By assuming that the IR emission is primarily caused by dust heating during star formation, the SFR remains proportional to the dust thermal emission of the galaxy. Kennicutt (1998) asserted that the total infrared luminosity $L_{\mathrm{IR}}$ emitted between 8 and $1000 \mu \mathrm{m}$ is a good tracer of the SFR. At the redshift of our interest $(z \sim 0.3-2.5)$, MIPS $24 \mu \mathrm{m}$ band probes the rest-frame mid-IR emission, which has been demonstrated to correlate with $L_{\mathrm{IR}}$ (e.g., Chary \& Elbaz 2001; Elbaz et al. 2002; Papovich \& Bell 2002; Takeuchi et al. 2005; Zhu et al. 2008).

Since dust emits most of its light at longer wavelengths, $L_{\mathrm{IR}}$ extrapolation from $24 \mu \mathrm{m}$ observations can be a difficult procedure. The rest-frame region under study is particularly complex given the presence of polycyclic aromatic hydrocarbon $(\mathrm{PAH})$ lines. In principle, these aspects could easily lead to nonnegligible errors in the determination of $L_{\mathrm{IR}}$ values. Therefore, we employed two different methods to infer $L_{\mathrm{IR}}$, examining and comparing both synthetic and empirical templates using our $K_{\mathrm{s}}$-selected subsample (subsample A).

Firstly, according to procedures normally adopted in the literature, we considered Dale \& Helou (2002, DH hereafter) and Chary \& Elbaz (2001, CE hereafter) synthetic libraries. These models do not extend to sufficiently short wavelengths to fit the SED shape, i.e., they do not include the stellar contribution, and at $z \gtrsim 1$ only MIPS $24 \mu \mathrm{m}$ band data can be fitted, the other bands moving out of the model range or being shifted to a wavelength region dominated by star light. Each model is associated with a 


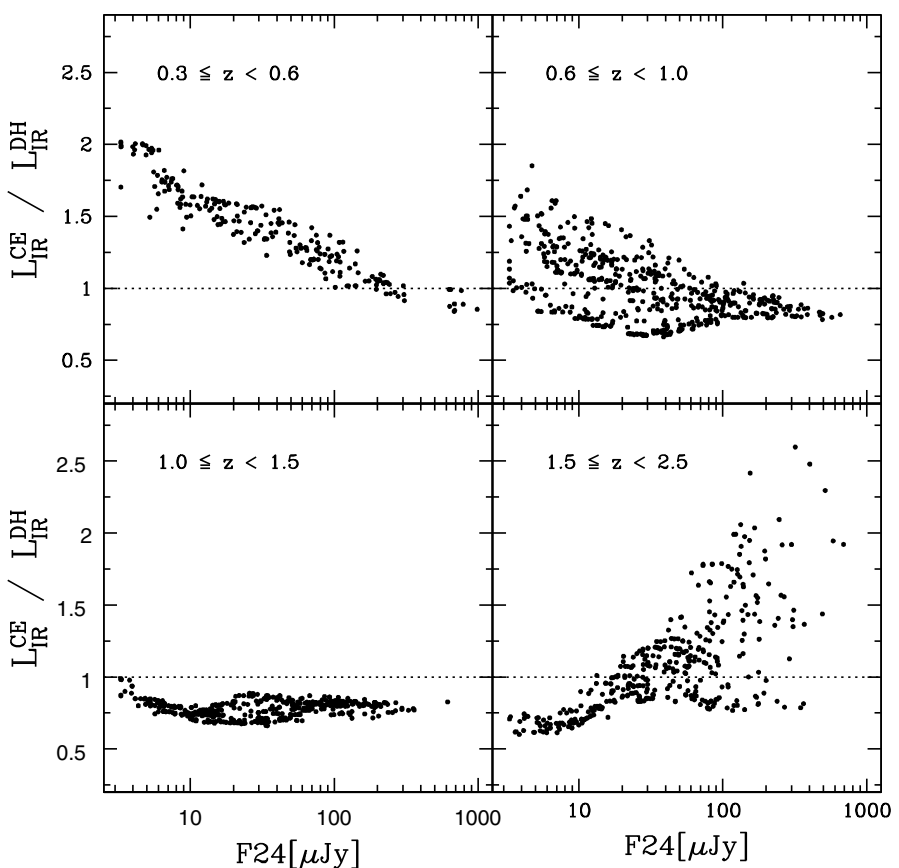

Fig. A.1. Comparison between the total infrared luminosity estimated with Dale \& Helou (2002) and Chary \& Elbaz (2001) templates in different redshift bins. The two synthetic libraries predictions are in good agreement within a factor $<3$.

given $L_{\mathrm{IR}}$. In the case of the CE library, each model is provided with its absolute normalization, and hence with a given total infrared luminosity. As for the DH library, we assigned a given $L_{\mathrm{IR}}$ to each template using the empirical relation in Marcillac et al. (2006) between $L_{\mathrm{IR}}$ and the predicted $f_{v}^{60} / f_{v}^{100}$ colour. In both cases and for each source, once the $k$-correction had been applied, we selected the model that reproduced the $24 \mu \mathrm{m}$ observed luminosity and normalized it using the flux difference between the model and the observed galaxy.

In Fig. A.1, we show a comparison between $L_{\mathrm{IR}}$ predicted by the two different model libraries. As already noticed in literature (Papovich et al. 2006; Marcillac et al. 2006), they give consistent results to within a factor 2-3, with the highest differences appearing at high redshifts and high luminosities. However, it is possible to observe a few trends with both luminosity and redshift that depend on the specific template details.

A new kind of approach in our work consists of employing empirical local spectra to fit the overall galaxy SED shape instead of the $24 \mu \mathrm{m}$ luminosity. These spectra include the stellar contribution as well as the effects of dust emission. We considered Polletta et al. (2007) spectra (POL hereafter). This library comprises early-type, spiral, starburst, and different kinds of AGN local templates, ranging from $\sim 100 \AA$ to $\sim 5 \mathrm{~mm}$. The wide wavelength extension allows us to fit the SED shape using the multiband catalog. After performing several tests, we decided to carry out a fit over 5 bands (IRAC bands $+24 \mu \mathrm{m}$ ), excluding the optical range, where the evolution in the stellar component has a more significant effect than the dust emission. Since we removed AGN-dominated objects from our sample, we only fit early-type and star-forming models. If we instead include AGN SEDs, the estimated $L_{\mathrm{IR}}$ is only poorly affected, resulting in slightly lower values (see also Papovich et al. 2007). Despite the poor statistical weight (one amongst five bands) and higher noise, the $24 \mu \mathrm{m}$ band is still fitted acceptably. The mean deviation between observed and fitted magnitudes for $24 \mu \mathrm{m}$ detections is $(-0.28 \pm 0.53) \mathrm{mag}, 68 \%$ of objects being consistent within 0.48 mag and $95 \%$ of objects being consistent within $1 \mathrm{mag}$ (considering the $F_{24}>20 \mu \mathrm{Jy}$ subsample). An example of different kinds of spectral energy distributions fitted by Polletta et al. (2007) templates is reported in Fig. A.2.

Figure A. 3 shows the comparison between $L_{\mathrm{IR}}$ estimates obtained with the DH synthetic library and the POL empirical one. Source classification based on the spectral shape fitting agrees with that assumed from $24 \mu \mathrm{m}$ emission. As expected, the only galaxies fitted by early-type SEDs (red crosses) have very faint $24 \mu \mathrm{m}$ emission. Moreover, objects tend to be fitted by starburst-like models (black dots) rather than spiral-like ones (gray dots) as redshift increases. Although the scatter between the two $L_{\mathrm{IR}}$ estimates is larger than in the previous case (note that, in contrast to Fig. A.1, the $y$-scale is logarithmic), we found global consistency between the two adopted procedures. The only relevant deviation affects the highest redshift bin: in this redshift range synthetic models appear to overpredict, by up to a factor of $10, L_{\mathrm{IR}}$ for bright objects. The same behaviour was observed by Papovich et al. (2007), where $24 \mu \mathrm{m}$ flux, fitted with DH library, seems to overestimate the SFR (which is almost proportional to $L_{\mathrm{IR}}$ ) with respect to the case where longer wavelengths (70 and $160 \mu \mathrm{m}$ MIPS bands) are considered as well. They corrected this trend using an empirical second-order polynomial. A similar trend of the $24 \mu \mathrm{m}$ flux overestimating $L_{\mathrm{IR}}$ at bright luminosities was also pointed out by Bavouzet et al. (2008) and Rieke et al. (2009). In our work, we used the Dale \& Helou (2002) library for consistency with previous works and for which the lowering correction needed to estimate the SFR was provided by Papovich et al. (2007).

In Fig. A.4, we show $L_{\mathrm{IR}}$, estimated using both synthetic (DH) and empirical (POL) templates, as a function of redshift. Once again, the two libraries provide fairly consistent results. All in all, the conversion between fluxes and total infrared luminosity does not depend on the assumed templates in a significant way. The observed $L_{\mathrm{IR}}$-redshift relation agrees with that presented by Pérez-González et al. (2005). As expected, we are only able to detect the more luminous sources as we move to higher redshift.

\section{Appendix B: Error analysis for $\boldsymbol{S} F \boldsymbol{R}_{\mathrm{fit}}$}

In Sect. 3.2, we compared two different tracers for the star formation rate, one derived from the $24 \mu \mathrm{m}$ emission and the other inferred from a SED fitting technique. Although the two estimators were found to be consistent overall, in a more careful analysis they were found to exhibit a systematic trend depending on the SFR itself as discussed in the text. To assess the reliability of the SFR measurements inferred from the SED fitting, and hence concrete evidence of this characteristic trend, we applied an error analysis similar to that widely adopted in similar cases (Papovich et al. 2001; Fontana et al. 2006, 2009). Briefly, the full synthetic library used to identify the best fit spectrum was compared with the observed SED of each galaxy. For each spectral model (i.e., for each combination of the free parameters age, $\tau, Z, E(B-V)$ ), the probability $P$ of the resulting $\chi^{2}$ was computed and retained, along with the associated SFR. In the case of galaxies with only photometric $z$, an additional source of error was the redshift uncertainty. To account for this, the error analysis was completed by allowing the redshift to be a free parameter at a local minimum around $z_{\text {phot }}$.

We chose two subsamples by performing the error analysis, for the cases $S F R_{\mathrm{IR}+\mathrm{UV}} \ll S F R_{\mathrm{fit}}$ and $S F R_{\mathrm{IR}+\mathrm{UV}} \gg S F R_{\mathrm{fit}}$. These two subsamples were called subsample A1 and subsample A2, 

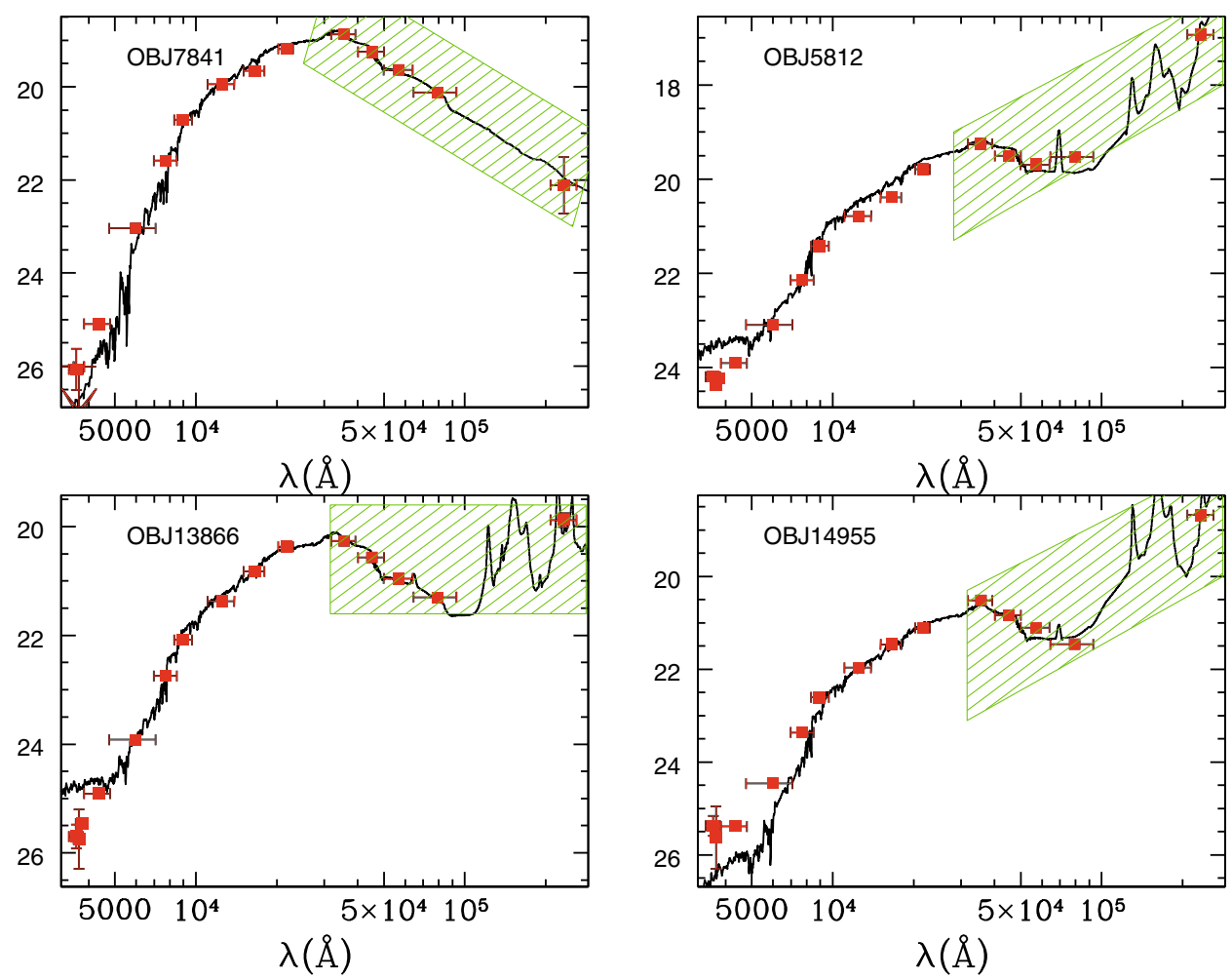

Fig. A.2. Different typical observed spectral energy distributions fitted by Polletta et al. (2007) templates. From top left, in anticlockwise direction, galaxies have been fitted by an elliptical, a spiral and two starbursts templates. The green shaded region indicates the wavelength range used for the fitting procedure.

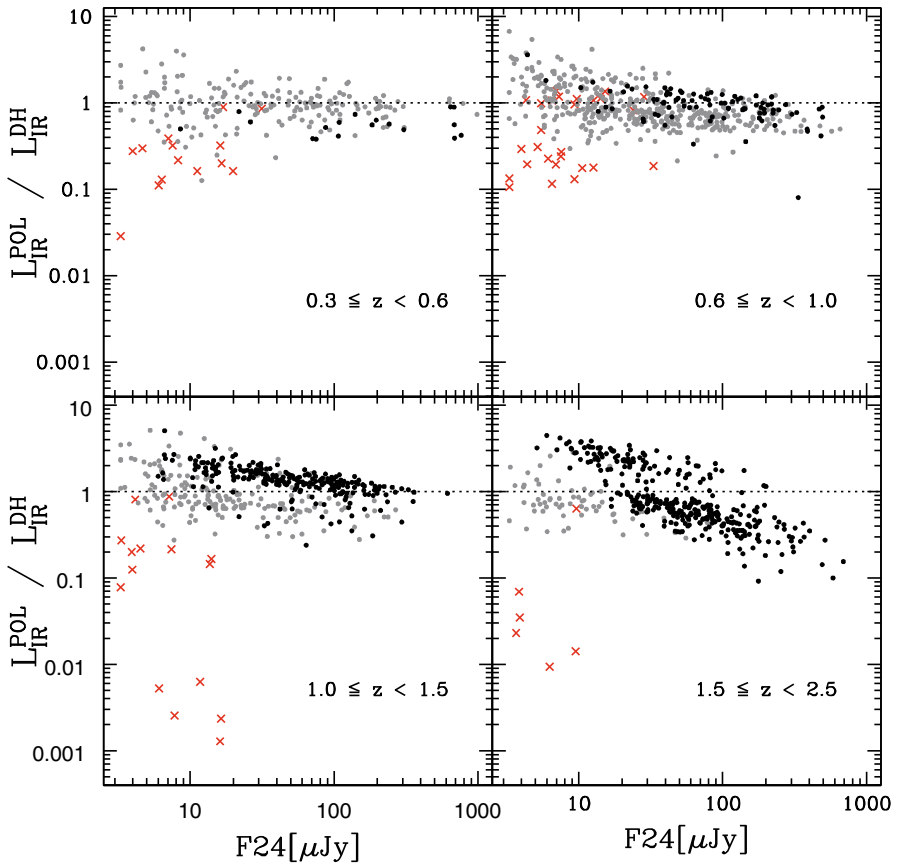

Fig. A.3. Comparison between the total infrared luminosity estimated with Dale \& Helou (2002) and Polletta et al. (2007) templates. Black and gray dots and red crosses indicate objects fitted respectively by starburst, spiral-like and early-type templates by Polletta et al. (2007). Synthetic models appear to overestimate $L_{\mathrm{IR}}$ at bright luminosities in the highest redshift bin when compared to empirical templates.

and selected to complete a reliable statistical analysis in both cases. Subsample A1 is made of galaxies having $0.6 \leq z<$ $1.0, S F R_{\mathrm{IR}+\mathrm{UV}}<10 M_{\odot} \mathrm{yr}^{-1}$ and $S F R_{\mathrm{IR}+\mathrm{UV}} / S F R_{\mathrm{fit}}<0.8$.

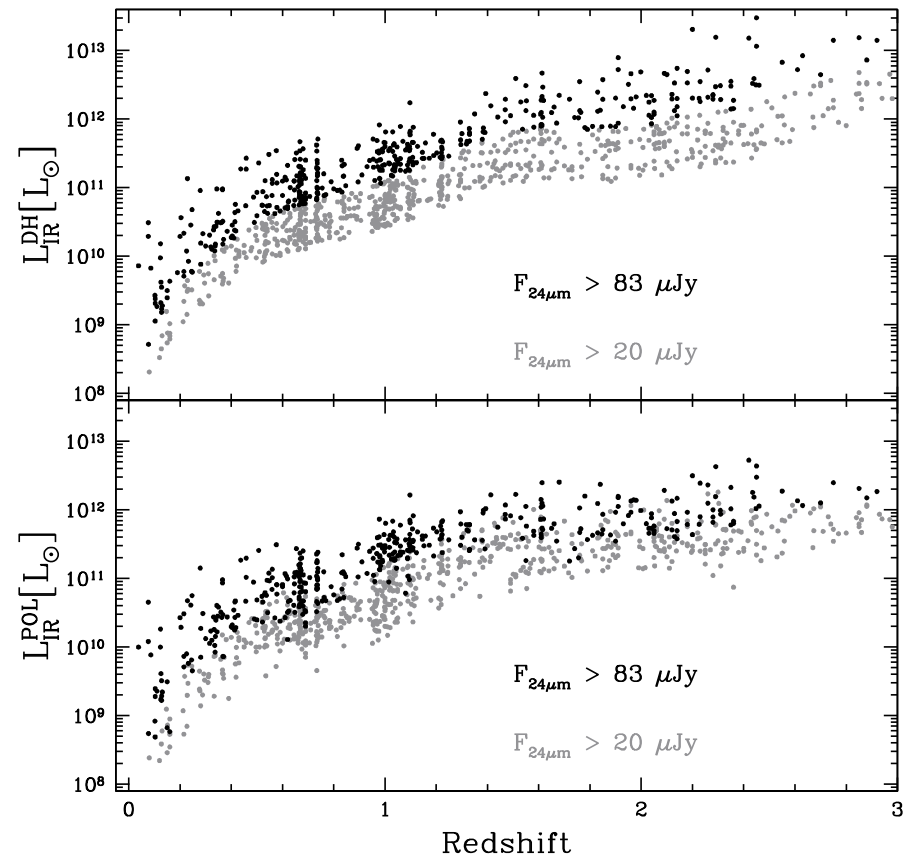

Fig. A.4. Relation between total infrared luminosity $L_{\mathrm{IR}}$, computed using Dale \& Helou (2002) (upper panel) and Polletta et al. (2007) templates (lower panel), and redshift. Black dots represent fluxes above $83 \mu \mathrm{Jy}$, gray + black dots refer to our sample limited at $20 \mu \mathrm{Jy}$.

Subsample A2 consists of galaxies in the redshift bin 1.5-2.5, with $S F R_{\mathrm{IR}+\mathrm{UV}}>200 M_{\odot} \mathrm{yr}^{-1}$ and $2<S F R_{\mathrm{IR}+\mathrm{UV}} / S F R_{\mathrm{fit}}<20$. Highly obscured AGN candidates were removed from both subsamples. 

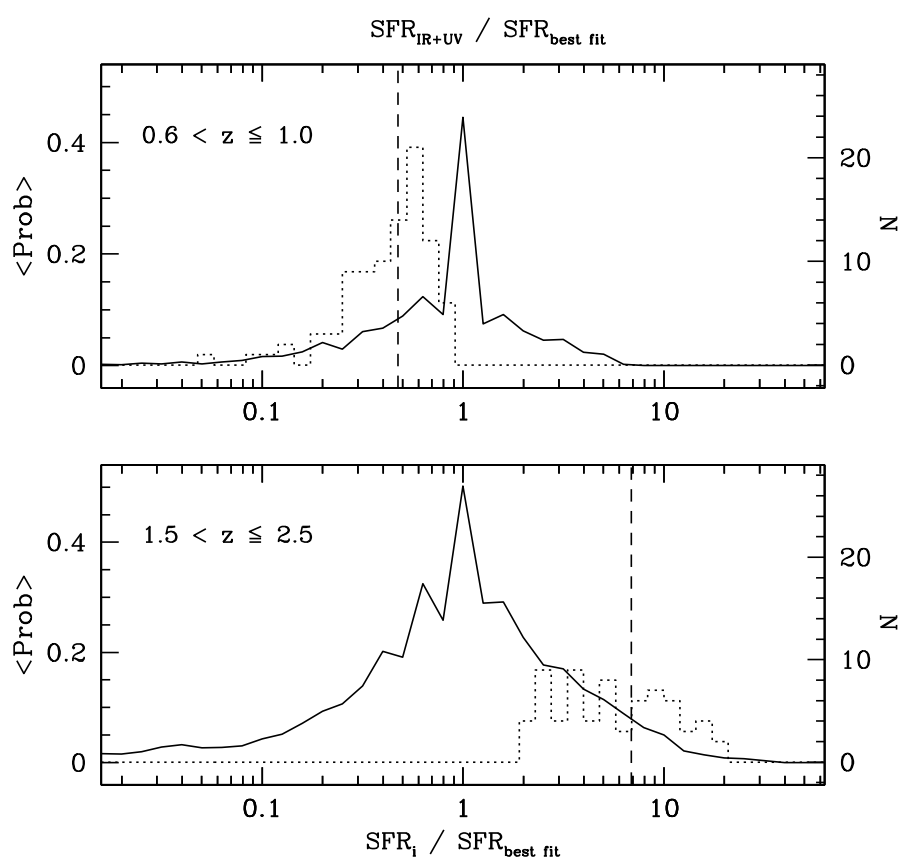

Fig. B.1. Probability distribution of the star formation rates estimated through the SED fitting technique averaged over each subsample. Upper and lower panels show subsample A1 and A2 (see text), respectively. The solid line shows the average probability associated with the SFR inferred from the generic fitted template, $S F R_{i}$, as a function of the ratio $S F R_{i} / S F R_{\text {best fit }}$. The dotted line shows the distribution of $S F R_{\mathrm{IR}+\mathrm{UV}} / S F R_{\text {best fit }}$ for all the galaxies in the subsample. The dashed line shows the average value for $S F R_{\mathrm{IR}+\mathrm{UV}} / S F R_{\text {best fit }}$.

The results of our analysis are shown in Fig. B.1. We show the probability distribution of the star formation rates estimated by the SED fitting averaged over each subsample. We plot the average probability associated to the SFR inferred from the generic fitted template, $S F R_{i}$, as a function of the ratio $S F R_{i} / S F R_{\text {best fit. }}$ We also show the distribution and the average value of $S F R_{\mathrm{IR}+\mathrm{UV}} / S F R_{\text {best fit }}$.

As expected, the probability curve is much wider at higher redshifts than at lower $z$, where the spread in our data also becomes larger (see Fig. 3). This is caused by the faintness of the galaxies observed at these redshifts and to the larger $k$-corrections. However, it is clear from Fig. B.1 that the SED fitting for galaxies in both subsamples is well constrained, and that the inferred average SFR is significantly higher (lower) than the one derived from the IR emission. Indeed, the average $S F R_{\mathrm{IR}+\mathrm{UV}} / S F R_{\text {best fit }}$ (dashed line) as well as their distribution (dotted line), occupy the tail of the $S F R_{i} / S F R_{\text {best fit }}$ distribution.

We also inspected the individual probability curves, considering, for each source, the ratio of the probability that the SFR from the SED fitting equals $S F R_{\mathrm{IR}+\mathrm{UV}}$ to the best-fit probability $P\left(S F R_{i}=S F R_{\mathrm{IR}+\mathrm{UV}}\right) / P\left(S F R_{i}=S F R_{\text {best fit }}\right)$. The fraction of galaxies with a ratio higher than 0.4 is $17.4 \%$ and $7.2 \%$ in subsamples A1 and A2, respectively.

Finally, according to a Kolmogorov-Smirnov test, the probability that the SFR values derived from the two tracers are drawn from the same distribution is negligible $\left(9.5 \times 10^{-15}\right.$ and $7.3 \times 10^{-20}$ for subsample A1 and subsample A2, respectively).

\section{References}

Adelberger, K. L., \& Steidel, C. C. 2000, ApJ, 544, 218

Baldry, I. K., \& Glazebrook, K. 2003, ApJ, 593, 258

Bavouzet, N., Dole, H., Le Floc'h, E., et al. 2008, A\&A, 479, 83

Bell, E. F. 2003, ApJ, 586, 794
Bell, E. F., Wolf, C., Meisenheimer, K., et al. 2004, ApJ, 608, 752 Bell, E. F., Papovich, C., Wolf, C., et al. 2005, ApJ, 625, 23 Bell, E. F., Zheng, X. Z., Papovich, C., et al. 2007, ApJ, 663, 834 Best, P. N., Kauffmann, G., Heckman, T. M., et al. 2005, MNRAS, 362, 25 Bower, R. G., Benson, A. J., Malbon, R., et al. 2006, MNRAS, 370, 645 Brinchmann, J., \& Ellis, R. S. 2000, ApJ, 536, L77

Brown, M. J. I., Dey, A., Jannuzi, B. T., et al. 2007, ApJ, 654, 858 Brusa, M., Fiore, F., Santini, P., et al. 2009, A\&A, submitted

Bruzual, A. G. 2007a, in IAU Symp. 241, ed. A. Vazdekis, \& R. F. Peletier, 125 Bruzual, G. 2007b, in From Stars to Galaxies: Building the Pieces to Build Up the Universe, ed. A. Vallenari, R. Tantalo, L. Portinari, \& A. Moretti, ASP Conf. Ser., 374, 303

Bruzual, G., \& Charlot, S. 2003, MNRAS, 344, 1000

Bundy, K., Ellis, R. S., Conselice, C. J., et al. 2006, ApJ, 651, 120

Calzetti, D. 1997, AJ, 113, 162

Calzetti, D. 2001, PASP, 113, 1449

Calzetti, D. 2008, in ASP Conf. Ser. 390, ed. J. H. Knapen, T. J. Mahoney, \& A. Vazdekis, 121

Calzetti, D., Kinney, A. L., \& Storchi-Bergmann, T. 1994, ApJ, 429, 582

Calzetti, D., Armus, L., Bohlin, R. C., et al. 2000, ApJ, 533, 682

Calzetti, D., Kennicutt, R. C., Engelbracht, C. W., et al. 2007, ApJ, 666, 870

Caputi, K. I., Lagache, G., Yan, L., et al. 2007, ApJ, 660, 97

Chabrier, G. 2003, ApJ, 586, L133

Chary, R., \& Elbaz, D. 2001, ApJ, 556, 562

Chary, R.-R. 2007, in Deepest Astronomical Surveys, ed. J. Afonso, H. C. Ferguson, B. Mobasher, \& R. Norris, ASP Conf. Ser., 380, 375 Cimatti, A., Cassata, P., Pozzetti, L., et al. 2008, A\&A, 482, 21 Cowie, L. L., Songaila, A., Hu, E. M., \& Cohen, J. G. 1996, AJ, 112, 839

Cresci, G., Hicks, E. K. S., Genzel, R., et al. 2009, ApJ, 697, 115

Croton, D. J., Springel, V., White, S. D. M., et al. 2006, MNRAS, 367, 864

Daddi, E., Cimatti, A., Renzini, A., et al. 2004, ApJ, 617, 746

Daddi, E., Alexander, D. M., Dickinson, M., et al. 2007a, ApJ, 670, 173

Daddi, E., Dickinson, M., Morrison, G., et al. 2007b, ApJ, 670, 156

Dale, D. A., \& Helou, G. 2002, ApJ, 576, 159

Damen, M., Labbé, I., Franx, M., et al. 2009, ApJ, 690, 937

Davé, R. 2008, MNRAS, 385, 147

De Lucia, G., \& Blaizot, J. 2007, MNRAS, 375, 2

De Santis, C., Grazian, A., Fontana, A., \& Santini, P. 2007, New Astron., 12, 271 Dekel, A., \& Birnboim, Y. 2006, MNRAS, 368, 2

Dekel, A., Birnboim, Y., Engel, G., et al. 2009, Nature, 457, 451

Dey, A., Soifer, B. T., Desai, V., et al. 2008, ApJ, 677, 943

Dickinson, M., Papovich, C., Ferguson, H. C., \& Budavári, T. 2003, ApJ, 587, 25

Drory, N., Bender, R., Feulner, G., et al. 2004, ApJ, 608, 742

Dunne, L., Ivison, R. J., Maddox, S., et al. 2009, MNRAS, 394, 3 Elbaz, D., Cesarsky, C. J., Chanial, P., et al. 2002, A\&A, 384, 848 Elbaz, D., Daddi, E., Le Borgne, D., et al. 2007, A\&A, 468, 33

Faber, S. M., Willmer, C. N. A., Wolf, C., et al. 2007, ApJ, 665, 265

Ferreras, I., Saha, P., \& Williams, L. L. R. 2005, ApJ, 623, L5

Feulner, G., Gabasch, A., Salvato, M., et al. 2005, ApJ, 633, L9

Fiore, F., Grazian, A., Santini, P., et al. 2008, ApJ, 672, 94

Fontana, A., Donnarumma, I., Vanzella, E., et al. 2003, ApJ, 594, L9

Fontana, A., Pozzetti, L., Donnarumma, I., et al. 2004, A\&A, 424, 23

Fontana, A., Salimbeni, S., Grazian, A., et al. 2006, A\&A, 459, 745

Fontana, A., Santini, P., Grazian, A., et al. 2009, A\&A, 501, 15

Fontanot, F., De Lucia, G., Monaco, P., Somerville, R. S., \& Santini, P. 2009, MNRAS, 397, 1776

Genel, S., Genzel, R., Bouché, N., et al. 2008, ApJ, 688, 789

Giavalisco, M., Ferguson, H. C., Koekemoer, A. M., et al. 2004, ApJ, 600, L93

Glazebrook, K., Abraham, R. G., McCarthy, P. J., et al. 2004, Nature, 430, 181

Grazian, A., Fontana, A., De Santis, C., et al. 2006, A\&A, 449, 951

Grazian, A., Salimbeni, S., Pentericci, L., et al. 2007, A\&A, 465, 393

Han, Z., Podsiadlowski, P., \& Lynas-Gray, A. E. 2007, MNRAS, 380, 1098

Heavens, A., Panter, B., Jimenez, R., \& Dunlop, J. 2004, Nature, 428, 625

Hopkins, A. M. 2004, ApJ, 615, 209

Hopkins, A. M., \& Beacom, J. F. 2006, ApJ, 651, 142

Iglesias-Páramo, J., Buat, V., Takeuchi, T. T., et al. 2006, ApJS, 164, 38

Johansson, P. H., Naab, T., \& Ostriker, J. P. 2009, ApJ, 697, L38

Juneau, S., Glazebrook, K., Crampton, D., et al. 2005, ApJ, 619, L135

Kennicutt, Jr., R. C. 1998, ARA\&A, 36, 189

Khochfar, S., \& Ostriker, J. P. 2008, ApJ, 680, 54

Khochfar, S., \& Silk, J. 2008, arXiv e-prints

Kitzbichler, M. G., \& White, S. D. M. 2007, MNRAS, 376, 2

Lagache, G., Dole, H., \& Puget, J.-L. 2003, MNRAS, 338, 555

Le Floc'h, E., Papovich, C., Dole, H., et al. 2005, ApJ, 632, 169

Lemson, G. \& Springel, V. 2006, in Astronomical Data Analysis Software and Systems XV, ed. C. Gabriel, C. Arviset, D. Ponz, \& S. Enrique, ASP Conf. Ser., 351,212 
Lilly, S. J., Le Fevre, O., Hammer, F., \& Crampton, D. 1996, ApJ, 460, L1 Lo Faro, B., Monaco, P., Vanzella, E., et al. 2009, MNRAS, accepted Madau, P., Ferguson, H. C., Dickinson, M. E., et al. 1996, MNRAS, 283, 1388 Maiolino, R., Nagao, T., Grazian, A., et al. 2008, A\&A, 488, 463 Maraston, C. 2005, MNRAS, 362, 799

Marchesini, D., van Dokkum, P. G., Forster Schreiber, N. M., et al. 2008, [arXiv:0811.1773]

Marcillac, D., Elbaz, D., Chary, R. R., et al. 2006, A\&A, 451, 57

Menci, N., Cavaliere, A., Fontana, A., et al. 2004, ApJ, 604, 12

Menci, N., Fontana, A., Giallongo, E., Grazian, A., \& Salimbeni, S. 2006, ApJ, 647,753

Monaco, P., Fontanot, F., \& Taffoni, G. 2007, MNRAS, 375, 1189

Nagamine, K., Ostriker, J. P., Fukugita, M., \& Cen, R. 2006, ApJ, 653, 881

Noeske, K. G., Weiner, B. J., Faber, S. M., et al. 2007, ApJ, 660, L43

Papovich, C., \& Bell, E. F. 2002, ApJ, 579, L1

Papovich, C., Dickinson, M., \& Ferguson, H. C. 2001, ApJ, 559, 620

Papovich, C., Dole, H., Egami, E., et al. 2004, ApJS, 154, 70

Papovich, C., Moustakas, L. A., Dickinson, M., et al. 2006, ApJ, 640, 92

Papovich, C., Rudnick, G., Le Floc'h, E., et al. 2007, ApJ, 668, 45

Pérez-González, P. G., Rieke, G. H., Egami, E., et al. 2005, ApJ, 630, 82

Polletta, M., Tajer, M., Maraschi, L., et al. 2007, ApJ, 663, 81

Popesso, P., Dickinson, M., Nonino, M., et al. 2009, A\&A, 494, 443

Pozzetti, L., Bolzonella, M., Lamareille, F., et al. 2007, A\&A, 474, 443

Rieke, G. H., Young, E. T., Engelbracht, C. W., et al. 2004, ApJSS, 154, 25

Rieke, G. H., Alonso-Herrero, A., Weiner, B. J., et al. 2009, ApJ, 692, 556
Rodighiero, G., Vaccari, M., Franceschini, A., et al. 2009, A\&A, submitted Rudnick, G., Labbé, I., Förster Schreiber, N. M., et al. 2006, ApJ, 650, 624 Salimbeni, S., Giallongo, E., Menci, N., et al. 2008, A\&A, 477, 763

Salimbeni, S., Fontana, A., Giallongo, E., et al. 2009, in AIP Conf. Ser. 1111 , ed. G. Giobbi, A. Tornambe, G. Raimondo, M. Limongi, L. A. Antonelli, N. Menci, \& E. Brocato, 207

Salpeter, E. E. 1955, ApJ, 121, 161

Shapley, A. E., Steidel, C. C., Adelberger, K. L., et al. 2001, ApJ, 562, 95

Shapley, A. E., Steidel, C. C., Erb, D. K., et al. 2005, ApJ, 626, 698

Shupe, D. L., Rowan-Robinson, M., Lonsdale, C. J., et al. 2008, AJ, 135, 1050 Siebenmorgen, R., \& Krügel, E. 2007, A\&A, 461, 445

Springel, V., White, S. D. M., Jenkins, A., et al. 2005, Nature, 435, 629

Steidel, C. C., Adelberger, K. L., Giavalisco, M., Dickinson, M., \& Pettini, M. 1999, The Astrophysical Journal, 519, 1

Takeuchi, T. T., Buat, V., Iglesias-Páramo, J., Boselli, A., \& Burgarella, D. 2005, A\&A, 432, 423

Thomas, D., Maraston, C., Bender, R., \& Mendes de Oliveira, C. 2005, ApJ, 621,673

Vanzella, E., Cristiani, S., Dickinson, M., et al. 2008, A\&A, 478, 83

Wilkins, S. M., Trentham, N., \& Hopkins, A. M. 2008, MNRAS, 385, 687

Wuyts, S., Labbé, I., Schreiber, N. M. F., et al. 2008, ApJ, 682, 985

Yan, H., Dickinson, M., Giavalisco, M., et al. 2006, ApJ, 651, 24

Zheng, X. Z., Bell, E. F., Papovich, C., et al. 2007, ApJ, 661, L41

Zhu, Y.-N., Wu, H., Cao, C., \& Li, H.-N. 2008, ApJ, 686, 155

Zucca, E., Ilbert, O., Bardelli, S., et al. 2006, A\&A, 455, 879 